\title{
A METHOD OF BIOLOGICAL TISSUES ELASTICITY RECONSTRUCTION USING MAGNETIC RESONANCE ELASTOGRAPHY MEASUREMENTS
}

\author{
BY \\ HABIB AMMARI (Laboratoire Ondes et Acoustique, CNRS ES ESPCI, 10 rue Vauquelin, 75231 \\ Paris Cedex 05, France), \\ PIERRE GARAPON (Laboratoire Ondes et Acoustique, CNRS \&S ESPCI, 10 rue Vauquelin, 75231 \\ Paris Cedex 05, France), \\ HYEONBAE KANG (School of Mathematical Sciences, Seoul National University, Seoul 151-747, \\ Korea), \\ AND
}

HYUNDAE LEE (School of Mathematical Sciences, Seoul National University, Seoul 151-747, Korea)

\begin{abstract}
Magnetic resonance elastography (MRE) is an approach to measuring material properties using external vibration in which the internal displacement measurements are made with magnetic resonance. A variety of simple methods have been designed to recover mechanical properties by inverting the displacement data. Currently, the remaining problems with all of these methods are that, in general, the homogeneous Helmholtz equation is used and therefore it fails at interfaces between tissues of different properties. The purpose of this work is to propose a new method for reconstructing both the shape and the shear modulus of a small anomaly with Lamé parameters different from the background ones using internal displacement measurements.
\end{abstract}

1. Introduction. Changes in tissue elasticity are generally correlated with pathological phenomena. Many cancers appear as extremely hard nodules.

\footnotetext{
Received March 26, 2007.

2000 Mathematics Subject Classification. Primary 35R30, 35B20; Secondary 74B05, 35Q30.

Key words and phrases. Elastic imaging, reconstruction, quasi-incompressible elasticity, layer potentials, Stokes system, small volume asymptotic expansions, level set method.

The first author was supported in part by the Brain Pool Korea Program at Seoul National University and by the project ANR-06-BLAN-0089.

The second and fourth authors were supported in part by the BK21 Math. division at Seoul National University.

The third author was supported in part by the grant KOSEF R01-2006-000-10002-0.

E-mail address: habib.ammari@polytechnique.fr

E-mail address: Pierre.Garapon@espci.fr

E-mail address: hbkang@snu.ac.kr

E-mail address: hdlee@math.snu.ac.kr
} 
Magnetic resonance elastography (MRE) is a recently developed technique that can directly visualize and quantitatively measure the displacement's field in tissues subject to harmonic mechanical excitation at low frequencies $(10$ to $1000 \mathrm{~Hz})$. A phase-contrast MRI technique is used to spatially map and measure the complete three-dimensional displacement patterns. From this data, local quantitative values of shear modulus can be calculated and images that depict tissue elasticity or stiffness can be generated. See $[31,29,20]$.

To recover the shear modulus from these images, some approximations are needed: the body is supposed to be linearly elastic, isotropic and quasi-incompressible. This last assumption is true for the biologic tissues. It is the root of both the numerical efficiency of the existing model and of its flaws in terms of precision and reliability. The method can be summarized in the following way: if $\mathbf{u}$ is the displacement field, $(\lambda, \mu)$ the Lamé coefficients and $\rho$ the material density, the elasticity system in a homogeneous medium reads

$$
\mu \Delta \mathbf{u}+(\lambda+\mu) \nabla \nabla \cdot \mathbf{u}=-\omega^{2} \rho \mathbf{u} .
$$

The solution to this equation can be split into two parts: $\mathbf{u}=\mathbf{u}_{S}+\mathbf{u}_{P} ;$ a null divergence solution $\mathbf{u}_{S}$ (shear waves $\mathrm{S}$ ) and an irrotational solution $\mathbf{u}_{P}$ (compression waves $\mathrm{P}$ ) having respective propagation speeds of $V_{S}=\sqrt{\mu / \rho}$ and $V_{P}=\sqrt{(\lambda+2 \mu) / \rho}$. These two waves interact via mode conversion at boundaries and interfaces. In soft tissues, quasi-incompressibility leads to $\lambda \gg \mu$ and thus the compression waves $\mathbf{u}_{P}$ propagate much faster than the shear waves $\mathbf{u}_{S}$. To remove $\lambda$ from consideration, approximations on the relative order of magnitude of the different terms involved in the elasticity system are performed; they may not be fully justified but result in a great simplification of the equations. In [29, 20], the elasticity system is reduced to three decoupled Helmholtz equations:

$$
\mu \Delta u_{i}=-\omega^{2} \rho u_{i}, \quad i=1,2,3, \quad \mathbf{u}=\left(u_{1}, u_{2}, u_{3}\right) .
$$

A very simple local inversion formula is obtained for the shear modulus $\mu$ :

$$
\mu=-\frac{\omega^{2} \rho u_{i}}{\Delta u_{i}}
$$

This formula is first smoothed by a Fourier transform and then used to reconstruct the shear modulus in nonhomogeneous media. Thus, measurements in only one sensitization direction (and an estimate of the Laplacian of that component) suffice to determine $\mu$. A slightly different method developed in [36] consists of applying the curl-operator to the measured displacement field $\mathbf{u}$ in order to completely remove contributions from the compressional wave $\mathbf{u}_{P}$.

This general and simple approach described in [29, 20] sounds attractive since it produces instantaneously an image out of the experimental device, without any need to solve a difficult, ill-posed, inverse problem. However, although it is showing images, it is still far from being able to produce a reliable examination tool. Part of the problems encountered come from technological enhancements still to come, but the most important flaws are more fundamental. They are due to the approximations briefly explained above that are not always valid, and are too strong to allow an accurate reconstruction of the map of the mechanical coefficients, in particular in the presence of anomalies. The 
existing results found in the literature are clearly not satisfactory and are in some cases far from giving an appropriate clinical answer, useful to physicians. It is not difficult to be convinced that the approximations performed above cause a dramatic loss in image resolution and image quality even though the signal-to-noise ratio for MRE measurements is very high. A more sophisticated and deeper mathematical analysis is certainly necessary to make good use of such high-resolution measurements.

In this paper we tackle the problem of shear modulus reconstruction in the following directions, without the basic assumptions of the existing models concerning isotropy, incompressibility and relative magnitude of the various terms.

Firstly, we prove that the elasticity system in biological tissues can be replaced with a sequence of nonhomogeneous modified Stokes systems.

Secondly, we derive the leading-order term in the displacement field perturbations that are due to the presence of a small volume elastic anomaly. The method of small volume expansions has proven very useful in many other contexts. Such asymptotics have been investigated in the case of the conduction equation $[19,15,12,2,14]$, the operator of elasticity [1, 7], the Helmholtz equation or the Maxwell system [39, 9, 4, 8]. See also the book [3] and its list of references. The remarkable feature of this technique is that it allows a stable and accurate reconstruction of the location and of the geometric features of the anomalies, even for moderately noisy data.

Thirdly, we combine the inverse problem techniques for small anomalies and a level set algorithm to solve the full three-dimensional inverse problem of the MRE. The method of asymptotic expansions of small volume anomalies plays a central role in improving shear modulus reconstruction.

The paper is organized as follows. In the next section, we describe the physical model problem. Section 3 is devoted to a rigorous derivation of an asymptotic formula of the displacement field in the presence of an elastic anomaly as the parameters $\lambda$ and $\tilde{\lambda}$ of the background and the anomaly respectively go to $+\infty$ with $\tilde{\lambda} / \lambda=O(1)$. We prove that the elasticity system can be replaced with a sequence of nonhomogeneous modified Stokes systems. In Section 4, we describe a layer potential technique for solving the transmission problem for the modified Stokes system. The method of asymptotic expansions of small volume anomalies is introduced in Section 5. A rigorous derivation of the leading-order term in the asymptotic expansion of the displacement field as the volume of the elastic anomaly goes to zero is provided. A justification of this formula is based on a layer potential technique. The concept of viscous moment tensor (VMT) is introduced and its connection with the elastic moment tensor is highlighted. It is important from an imaging perspective to precisely characterize the VMT and derive bounds on its elements in order to develop efficient algorithms to reconstruct anomalies of small volume. In Section 6, based on the asymptotic expansion of the perturbations in the displacement field that are due to the elastic anomaly, we propose a new reconstruction method of level set type. It is expected that our algorithm is very effective for reconstructing both the shape and the shear modulus of the anomaly with a high resolution. Extensions to imaging of anisotropic anomalies as well as to transient elastography are given in Section 7 . The paper ends with a short discussion. 
It is worth emphasizing that although we deal only with real shear modulus, all results in this paper work for complex shear modulus with positive imaginary part (the shear viscosity) accounting for attenuation within the medium.

2. Model problem. We suppose that an elastic medium occupies a bounded domain $\Omega$ in $\mathbb{R}^{3}$, with a connected $\mathcal{C}^{2}$-boundary $\partial \Omega$. Let the constants $(\lambda, \mu)$ denote the Lamé coefficients of $\Omega$ that are the elastic parameters in the absence of any anomalies, and let the constant $\rho$ denote the density of the background. Suppose that $\Omega$ contains an elastic anomaly $D$ given by $D=\epsilon B+z$, where $B$ is a bounded $\mathcal{C}^{2}$-domain in $\mathbb{R}^{3}$. The domain $B$ is considered to be a reference domain, the small number $\epsilon$ is the diameter of $D$, and $z$ represents the location of $D$. We assume that there exists $c_{0}>0$ such that $\inf _{x \in D} \operatorname{dist}(x, \partial \Omega)>c_{0}$, which means that $D$ is away from the boundary $\partial \Omega$. Suppose that $D$ has a pair of Lamé constants $(\tilde{\lambda}, \tilde{\mu})$ which is different from that of the background elastic body, $(\lambda, \mu)$, and let $\tilde{\rho}$ denote its density. It is always assumed that

$$
\rho>0, \mu>0,3 \lambda+2 \mu>0, \quad \tilde{\rho}>0, \tilde{\mu}>0, \text { and } 3 \tilde{\lambda}+2 \tilde{\mu}>0 .
$$

Consider the following transmission problem associated to the system of elastodynamics with the Dirichlet boundary condition:

$$
\left\{\begin{array}{l}
\sum_{j, k, l=1}^{3} \frac{\partial}{\partial x_{j}}\left(C_{i j k l} \frac{\partial u_{k}}{\partial x_{l}}\right)+\omega^{2}(\rho \chi(\Omega \backslash D)+\tilde{\rho} \chi(D)) u_{i}=0 \quad \text { in } \Omega, \quad i=1,2,3, \\
\left.\mathbf{u}\right|_{\partial \Omega}=\mathbf{g},
\end{array}\right.
$$

where the elasticity tensor $C=\left(C_{i j k l}\right)$ is given by

$$
\begin{aligned}
C_{i j k l}:=( & \lambda \chi(\Omega \backslash D)+\tilde{\lambda} \chi(D)) \delta_{i j} \delta_{k l} \\
& +(\mu \chi(\Omega \backslash D)+\tilde{\mu} \chi(D))\left(\delta_{i k} \delta_{j l}+\delta_{i l} \delta_{j k}\right),
\end{aligned}
$$

$\omega>0$ is the angular frequency of the mechanical oscillations, and $u_{i}$ for $i=1,2,3$, denote the components of the displacement field $\mathbf{u}$. Here and throughout this paper $\delta_{i j}$ denotes the Kronecker's delta.

The elastostatic system corresponding to the Lamé constants $\lambda, \mu$ is defined by

$$
\mathcal{L}_{\lambda, \mu} \mathbf{u}:=\mu \Delta \mathbf{u}+(\lambda+\mu) \nabla \nabla \cdot \mathbf{u}
$$

The corresponding conormal derivative $\partial \mathbf{u} / \partial \nu$ on $\partial D$ is defined to be

$$
\frac{\partial \mathbf{u}}{\partial \nu}:=\lambda(\nabla \cdot \mathbf{u}) \mathbf{N}+\mu\left(\nabla \mathbf{u}+\nabla \mathbf{u}^{T}\right) \mathbf{N} \quad \text { on } \partial D
$$

where $\mathbf{N}=\left(N_{1}, N_{2}, N_{3}\right)$ is the outward unit normal to $\partial D$ and the superscript $T$ denotes the transpose of a matrix. Let $\mathcal{L}_{\tilde{\lambda}, \tilde{\mu}}$ and $\partial / \partial \tilde{\nu}$ be the Lamé system and the conormal derivative associated with $(\tilde{\lambda}, \tilde{\mu})$, respectively. Then $(2.2)$ is equivalent to the following 
problem:

$$
\left\{\begin{array}{l}
\mathcal{L}_{\lambda, \mu} \mathbf{u}+\omega^{2} \rho \mathbf{u}=0 \quad \text { in } \Omega \backslash \bar{D} \\
\mathcal{L}_{\tilde{\lambda}, \tilde{\mu}} \mathbf{u}+\omega^{2} \tilde{\rho} \mathbf{u}=0 \quad \text { in } D \\
\left.\mathbf{u}\right|_{-}=\left.\mathbf{u}\right|_{+} \text {on } \partial D \\
\left.\frac{\partial \mathbf{u}}{\partial \tilde{\nu}}\right|_{-}=\left.\frac{\partial \mathbf{u}}{\partial \nu}\right|_{+} \quad \text { on } \partial D \\
\left.\mathbf{u}\right|_{\partial \Omega}=\mathbf{g} .
\end{array}\right.
$$

Here and throughout this paper the subscripts \pm denote the limit from outside and inside $D$, respectively.

The inverse problem for MRE is to determine the shape and the elastic parameters of the anomaly $D$ from internal measurements of the displacement field $\mathbf{u}$. In most cases, the most significant elastic parameter to detect is the stiffness coefficient $\tilde{\mu}$.

The Poisson ratios $\sigma$ and $\tilde{\sigma}$ of the background and the anomaly are given in terms of the Lamé parameters by

$$
\sigma=\frac{\lambda / \mu}{1+2 \lambda / \mu} \quad \text { and } \quad \tilde{\sigma}=\frac{\tilde{\lambda} / \tilde{\mu}}{1+2 \tilde{\lambda} / \tilde{\mu}}
$$

It is known that in soft tissues, $\sigma, \tilde{\sigma} \approx 1 / 2$, or equivalently, $\lambda \gg \mu$ and $\tilde{\lambda} \gg \tilde{\mu}[20,29]$. This makes it difficult to estimate both parameters $\tilde{\mu}$ and $\tilde{\lambda}$ simultaneously.

3. Nearly incompressible materials. In this section we establish an asymptotic development of the solution to (2.6) as $\lambda$ and $\tilde{\lambda}$ go to $+\infty$ with $\tilde{\lambda} / \lambda=O(1)$. Assume for the sake of simplicity that $\int_{\partial \Omega} \mathbf{g} \cdot \mathbf{N}=0$. We show that the displacement field $\mathbf{u}$ can be represented in the form of a power series:

$$
\mathbf{u}=\mathbf{u}_{0}+\left(\frac{1}{\lambda} \chi(\Omega \backslash D)+\frac{1}{\tilde{\lambda}} \chi(D)\right) \mathbf{u}_{1}+\left(\frac{1}{\lambda^{2}} \chi(\Omega \backslash D)+\frac{1}{\tilde{\lambda}^{2}} \chi(D)\right) \mathbf{u}_{2}+\ldots
$$

where $\mathbf{u}_{i}$ for $i=0,1, \ldots$, are solutions to modified Stokes systems, the one used for computing the leading-order term $\mathbf{u}_{0}$ being homogeneous. Following [26], we prove that this asymptotic series strongly converges in an appropriate Sobolev space.

Set

$$
p:= \begin{cases}\lambda \nabla \cdot \mathbf{u} & \text { in } \Omega \backslash \bar{D} \\ \tilde{\lambda} \nabla \cdot \mathbf{u} & \text { in } D\end{cases}
$$


and rewrite (2.6) in the following form:

$$
\left\{\begin{array}{l}
\mu \Delta \mathbf{u}+\left(1+\frac{\mu}{\lambda}\right) \nabla p+\omega^{2} \rho \mathbf{u}=0 \quad \text { in } \Omega \backslash \bar{D}, \\
\tilde{\mu} \Delta \mathbf{u}+\left(1+\frac{\tilde{\mu}}{\tilde{\lambda}}\right) \nabla p+\omega^{2} \tilde{\rho} \mathbf{u}=0 \quad \text { in } D, \\
\left.\mathbf{u}\right|_{-}=\left.\mathbf{u}\right|_{+} \text {on } \partial D, \\
\left.\left(p \mathbf{N}+\tilde{\mu} \frac{\partial \mathbf{u}}{\partial \mathbf{N}}\right)\right|_{-}=\left.\left(p \mathbf{N}+\mu \frac{\partial \mathbf{u}}{\partial \mathbf{N}}\right)\right|_{+} \quad \text { on } \partial D, \\
\left.\mathbf{u}\right|_{\partial \Omega}=\mathbf{g},
\end{array}\right.
$$

where, by abuse of notation, we set

$$
\frac{\partial \mathbf{u}}{\partial \mathbf{N}}:=\left(\nabla \mathbf{u}+\nabla \mathbf{u}^{T}\right) \mathbf{N}
$$

We look for a solution of (3.2) in the form of power series

$$
\left\{\begin{array}{l}
\mathbf{u}=\mathbf{u}_{0}+\left(\frac{1}{\lambda} \chi(\Omega \backslash D)+\frac{1}{\tilde{\lambda}} \chi(D)\right) \mathbf{u}_{1}+\left(\frac{1}{\lambda^{2}} \chi(\Omega \backslash D)+\frac{1}{\tilde{\lambda}^{2}} \chi(D)\right) \mathbf{u}_{2}+\ldots \\
p=p_{0}+\left(\frac{1}{\lambda} \chi(\Omega \backslash D)+\frac{1}{\tilde{\lambda}} \chi(D)\right) p_{1}+\left(\frac{1}{\lambda^{2}} \chi(\Omega \backslash D)+\frac{1}{\tilde{\lambda}^{2}} \chi(D)\right) p_{2}+\ldots
\end{array}\right.
$$

This leads to the recurrence relations

$$
\left\{\begin{array}{l}
\left(\mu \Delta+\omega^{2} \rho\right) \mathbf{u}_{0}+\nabla p_{0}=0 \text { in } \Omega \backslash \bar{D}, \\
\left(\tilde{\mu} \Delta+\omega^{2} \tilde{\rho}\right) \mathbf{u}_{0}+\nabla p_{0}=0 \text { in } D, \\
\left.\mathbf{u}_{0}\right|_{-}=\left.\mathbf{u}_{0}\right|_{+} \text {on } \partial D, \\
\left(\left.p_{0}\right|_{+}-\left.p_{0}\right|_{-}\right) \mathbf{N}+\left.\mu \frac{\partial \mathbf{u}_{0}}{\partial \mathbf{N}}\right|_{+}-\left.\tilde{\mu} \frac{\partial \mathbf{u}_{0}}{\partial \mathbf{N}}\right|_{-}=0 \text { on } \partial D, \\
\nabla \cdot \mathbf{u}_{0}=0 \text { in } \Omega, \\
\mathbf{u}_{0}=\mathbf{g} \text { on } \partial \Omega, \\
\int_{\Omega} p_{0}=0,
\end{array}\right.
$$


and, for $j \geq 1$,

$$
\left\{\begin{array}{l}
\left(\mu \Delta+\omega^{2} \rho\right) \mathbf{u}_{j}+\nabla p_{j}+\mu \nabla p_{j-1}=0 \quad \text { in } \Omega \backslash \bar{D} \\
\left(\tilde{\mu} \Delta+\omega^{2} \tilde{\rho}\right) \mathbf{u}_{j}+\nabla p_{j}+\tilde{\mu} \nabla p_{j-1}=0 \quad \text { in } D \\
\left.\mathbf{u}_{j}\right|_{-}=\left.\left(\frac{\tilde{\lambda}}{\lambda}\right)^{j} \mathbf{u}_{j}\right|_{+} \quad \text { on } \partial D \\
\left(\frac{\tilde{\lambda}}{\lambda}\right)^{j}\left(\left.p_{j}\right|_{+} \mathbf{N}+\left.\mu \frac{\partial \mathbf{u}_{j}}{\partial \mathbf{N}}\right|_{+}\right)-\left(\left.p_{j}\right|_{-} \mathbf{N}+\left.\tilde{\mu} \frac{\partial \mathbf{u}_{j}}{\partial \mathbf{N}}\right|_{-}\right)=0 \quad \text { on } \partial D \\
\nabla \cdot \mathbf{u}_{j}=p_{j-1} \quad \text { in } \Omega \\
\mathbf{u}_{j}=0 \quad \text { on } \partial \Omega \\
\int_{\Omega} p_{j}=0 .
\end{array}\right.
$$

Equations (3.4) are the time-harmonic linearized equations of incompressible fluids or the modified Stokes system. Equations (3.5) are nonhomogeneous. The assumption $\int_{\partial \Omega} \mathbf{g} \cdot \mathbf{N}=0$ enters in (3.4) as a compatibility condition. The fifth condition in (3.4) and (3.5) comes from the definition (3.1) of $p$. Moreover, we should notice that the leading-order term $\mathbf{u}_{0}$ is independent of $\lambda$ and $\tilde{\lambda}$, while higher-order terms do depend on $\tilde{\lambda} / \lambda$. This would suggest that by using a very sensitive measurement instrument it may be possible to extract this contrast from internal measurements of the displacement field.

In this next section we prove, using layer potential techniques, existence and uniqueness of a solution to equations (3.4) and (3.5) provided that $\omega^{2} \rho / \mu$ is not a Dirichlet eigenvalue of the Stokes system in either $D$ or $\Omega$.

Let $H^{1}(\Omega)$ denote the set of functions $\mathbf{v} \in L^{2}(\Omega)$ such that $\nabla \mathbf{v} \in L^{2}(\Omega)$ and let

$$
H_{0}^{1}(\Omega)=\left\{\mathbf{v} \in H^{1}(\Omega): \mathbf{v}=0 \text { on } \partial \Omega\right\} .
$$

We also introduce $H^{-1}(\Omega)=\left(H_{0}^{1}(\Omega)\right)^{*}$ and $H^{-1 / 2}(\partial \Omega)=\left(H^{1 / 2}(\partial \Omega)\right)^{*}$, where $H^{1 / 2}(\partial \Omega)$ is the standard trace space. Finally, set $\left(T_{1}, T_{2}\right)$ to be an orthonormal basis for the tangent plane to $\partial \Omega$ at $x$, and let $\partial / \partial T=\sum_{p=1}^{2}\left(\partial / \partial T_{p}\right) T_{p}$ denote the tangential derivative on $\partial \Omega$. We say that $\mathbf{u} \in H^{1}(\partial \Omega)$ if $\mathbf{u} \in L^{2}(\partial \Omega)$ and $\partial \mathbf{u} / \partial T \in L^{2}(\partial \Omega)$.

The following is our main result in this section.

TheOrem 3.1. Suppose that $\omega^{2}$ is not an eigenvalue of the transmission problem

$$
\left\{\begin{array}{l}
\mu \Delta \mathbf{v}+\omega^{2} \rho \mathbf{v}+\nabla q=0 \quad \text { in } \Omega \backslash \bar{D} \\
\tilde{\mu} \Delta \mathbf{v}+\omega^{2} \tilde{\rho} \mathbf{v}+\nabla q=0 \quad \text { in } D \\
\left.\mathbf{v}\right|_{-}=\left.\mathbf{v}\right|_{+} \quad \text { on } \partial D \\
\left.\left(q \mathbf{N}+\tilde{\mu} \frac{\partial \mathbf{v}}{\partial \mathbf{N}}\right)\right|_{-}=\left.\left(q \mathbf{N}+\mu \frac{\partial \mathbf{v}}{\partial \mathbf{N}}\right)\right|_{+} \text {on } \partial D \\
\nabla \cdot \mathbf{v}=0 \\
\left.\mathbf{v}\right|_{\partial \Omega}=0
\end{array}\right.
$$


There exists a positive constant $C$ independent of $\lambda$ and $\tilde{\lambda}$ such that the following error estimate holds for $\lambda$ and $\tilde{\lambda}$ large enough and for all integers $J$ :

$$
\left\|\mathbf{u}-\sum_{j=0}^{J}\left(\frac{1}{\lambda^{j}} \chi(\Omega \backslash D)+\frac{1}{\tilde{\lambda}^{j}} \chi(D)\right) \mathbf{u}_{j}\right\|_{H^{1}(\Omega)} \leq C\left(\frac{1}{\lambda^{J+\frac{1}{2}}}+\frac{1}{\tilde{\lambda}^{J+\frac{1}{2}}}\right) .
$$

Proof. (i) We first prove that if $\omega^{2}$ is not an eigenvalue of (3.6), then for $\lambda$ and $\tilde{\lambda}$ large enough it is not an eigenvalue of the transmission problem

$$
\left\{\begin{array}{l}
\mathcal{L}_{\lambda, \mu} \mathbf{v}+\omega^{2} \rho \mathbf{v}=0 \quad \text { in } \Omega \backslash \bar{D} \\
\mathcal{L}_{\tilde{\lambda}, \tilde{\mu}} \mathbf{v}+\omega^{2} \tilde{\rho} \mathbf{v}=0 \quad \text { in } D \\
\left.\mathbf{v}\right|_{-}=\left.\mathbf{v}\right|_{+} \text {on } \partial D \\
\left.\frac{\partial \mathbf{v}}{\partial \tilde{\nu}}\right|_{-}=\left.\frac{\partial \mathbf{v}}{\partial \nu}\right|_{+} \quad \text { on } \partial D \\
\left.\mathbf{v}\right|_{\partial \Omega}=0
\end{array}\right.
$$

For doing this, suppose that $\omega^{2}$ is an eigenvalue of (3.8) and let $\mathbf{v}$ be the corresponding eigenvector normalized in $L^{2}(\Omega)$. Integrating by parts yields the energy identity

$$
\begin{aligned}
& \int_{\Omega}(\lambda \chi(\Omega \backslash D)+\tilde{\lambda} \chi(D))|\nabla \cdot \mathbf{v}|^{2}+\frac{1}{2} \int_{\Omega}(\mu \chi(\Omega \backslash D)+\tilde{\mu} \chi(D))\left|\nabla \mathbf{v}+(\nabla \mathbf{v})^{T}\right|^{2} \\
& \quad=\omega^{2} \int_{\Omega}(\rho \chi(\Omega \backslash D)+\tilde{\rho} \chi(D))|\mathbf{v}|^{2},
\end{aligned}
$$

where $T$ denotes the transpose of a matrix. Since $\mathbf{v}$ is normalized, it follows in particular that

$$
\int_{\Omega \backslash D}|\nabla \cdot \mathbf{v}|^{2} d x \leq \frac{\omega^{2}(\rho+\tilde{\rho})}{\lambda} \text { and } \int_{D}|\nabla \cdot \mathbf{v}|^{2} d x \leq \frac{\omega^{2}(\rho+\tilde{\rho})}{\tilde{\lambda}} .
$$

If we put $q:=(\lambda \chi(\Omega \backslash D)+\tilde{\lambda} \chi(D)) \nabla \cdot \mathbf{v}$, then the following holds:

$$
\left\{\begin{array}{l}
\mu \Delta \mathbf{v}+\omega^{2} \rho \mathbf{v}+\nabla q=O\left(\frac{1}{\sqrt{\lambda}}\right) \quad \text { in } \Omega \backslash \bar{D}, \\
\tilde{\mu} \Delta \mathbf{v}+\omega^{2} \tilde{\rho} \mathbf{v}+\nabla q=O\left(\frac{1}{\sqrt{\tilde{\lambda}}}\right) \quad \text { in } D \\
\left.\mathbf{v}\right|_{-}=\left.\mathbf{v}\right|_{+} \text {on } \partial D, \\
\left.\left(q \mathbf{N}+\tilde{\mu} \frac{\partial \mathbf{v}}{\partial \mathbf{N}}\right)\right|_{-}=\left.\left(q \mathbf{N}+\mu \frac{\partial \mathbf{v}}{\partial \mathbf{N}}\right)\right|_{+} \quad \text { on } \partial D, \\
\nabla \cdot \mathbf{v}=O\left(\frac{1}{\sqrt{\lambda}}+\frac{1}{\sqrt{\tilde{\lambda}}}\right), \\
\left.\mathbf{v}\right|_{\partial \Omega}=0
\end{array}\right.
$$

provided that $\omega^{2}(\rho+\tilde{\rho})$ is bounded. Here the remainders $O\left(\frac{1}{\sqrt{\lambda}}\right)$ and $O\left(\frac{1}{\sqrt{\tilde{\lambda}}}\right)$ in the first two equations are in $H^{-1}(\Omega)$, while the one in the equation on the divergence is in $L^{2}(\Omega)$. Since $\omega^{2}$ is not an eigenvalue (3.6), it follows from the standard elliptic regularity theory 
that there exists a constant $C$ that may depend on $\omega$ but is independent of $\lambda, \tilde{\lambda}$, such that

$$
\|\mathbf{v}\|_{L^{2}(\Omega)} \leq C\left(\frac{1}{\sqrt{\lambda}}+\frac{1}{\sqrt{\tilde{\lambda}}}\right) .
$$

Since $\|\mathbf{v}\|_{L^{2}(\Omega)}=1$, which is impossible if $\lambda$ and $\tilde{\lambda}$ are large enough, we conclude that $\omega^{2}$ is not an eigenvalue of (3.8).

(ii) Define $\mathbf{e}_{J}$ in $\Omega$ by

$$
\mathbf{e}_{J}=\mathbf{u}-\sum_{j=0}^{J}\left(\frac{1}{\lambda^{j}} \chi(\Omega \backslash D)+\frac{1}{\tilde{\lambda}^{j}} \chi(D)\right) \mathbf{u}_{j} .
$$

By using (3.4) and (3.5) it follows that

$$
\left\{\begin{array}{l}
\mathcal{L}_{\lambda, \mu} \mathbf{e}_{J}+\omega^{2} \rho \mathbf{e}_{J}=\frac{1}{\lambda^{J}} \nabla p_{J} \quad \text { in } \Omega \backslash \bar{D} \\
\mathcal{L}_{\tilde{\lambda}, \tilde{\mu}} \mathbf{e}_{J}+\omega^{2} \tilde{\rho} \mathbf{e}_{J}=\frac{1}{\tilde{\lambda}^{J}} \nabla p_{J} \quad \text { in } D \\
\left.\mathbf{e}_{J}\right|_{+}=\left.\mathbf{e}_{J}\right|_{-} \text {on } \partial D \\
\left.\frac{\partial \mathbf{e}_{J}}{\partial \nu}\right|_{+}=\left.\frac{\partial \mathbf{e}_{J}}{\partial \tilde{\nu}}\right|_{-}+\left(\left.\frac{1}{\lambda^{J}} p_{J}\right|_{+}-\left.\frac{1}{\tilde{\lambda}^{J}} p_{J}\right|_{-}\right) \mathbf{N} \quad \text { on } \partial D \\
\left.\mathbf{e}_{J}\right|_{\partial \Omega}=0
\end{array}\right.
$$

We once again obtain the following energy identity by integration by parts:

$$
\begin{gathered}
\int_{\Omega}(\lambda \chi(\Omega \backslash D)+\tilde{\lambda} \chi(D))\left|\nabla \cdot \mathbf{e}_{J}\right|^{2}+\frac{1}{2} \int_{\Omega}(\mu \chi(\Omega \backslash D)+\tilde{\mu} \chi(D))\left|\nabla \mathbf{e}_{J}+\left(\nabla \mathbf{e}_{J}\right)^{T}\right|^{2} \\
-\omega^{2} \int_{\Omega}(\rho \chi(\Omega \backslash D)+\tilde{\rho} \chi(D))\left|\mathbf{e}_{J}\right|^{2}=\int_{\Omega}\left(\frac{1}{\lambda^{J}} \chi(\Omega \backslash D)+\frac{1}{\tilde{\lambda}^{J}} \chi(D)\right) p_{J} \nabla \cdot \mathbf{e}_{J} .
\end{gathered}
$$

We define the bilinear form $a(\cdot, \cdot)$ on $H_{0}^{1}(\Omega)$ by

$$
a(\mathbf{v}, \mathbf{v})=\int_{\Omega}(\lambda \chi(\Omega \backslash D)+\tilde{\lambda} \chi(D))|\nabla \cdot \mathbf{v}|^{2}+\frac{1}{2} \int_{\Omega}(\mu \chi(\Omega \backslash D)+\tilde{\mu} \chi(D))\left|\nabla \mathbf{v}+(\nabla \mathbf{v})^{T}\right|^{2}
$$

Then it follows that

$$
a\left(\mathbf{e}_{J}, \mathbf{e}_{J}\right)-\omega^{2} \int_{\Omega}(\rho \chi(\Omega \backslash D)+\tilde{\rho} \chi(D))\left|\mathbf{e}_{J}\right|^{2} \leq C\left(\frac{1}{\lambda^{J}}+\frac{1}{\tilde{\lambda}^{J}}\right)\left\|\nabla \cdot \mathbf{e}_{J}\right\|_{L^{2}(\Omega)}\left\|p_{J}\right\|_{L^{2}(\Omega)} .
$$

Applying Korn's inequality (see [6, Lemma 9.8]) yields that $a(\cdot, \cdot)$ is coercive. By the theory of collectively compact operators (see [10] or [8, Lemma 2.1]), we can see that if (3.7) holds for $\omega=0$, then it holds for any $\omega^{2}$ that is not an eigenvalue of (3.6), provided that $\lambda$ and $\tilde{\lambda}$ are sufficiently large.

(iii) We now prove (3.7) when $\omega=0$. Observe from (3.9) that

$$
a\left(\mathbf{e}_{J}, \mathbf{e}_{J}\right) \leq C\left(\frac{1}{\lambda^{J}}+\frac{1}{\tilde{\lambda}^{J}}\right)\left\|\nabla \cdot \mathbf{e}_{J}\right\|_{L^{2}(\Omega)},
$$

and hence

$$
\inf (\mu, \tilde{\mu})\left\|\mathbf{e}_{J}\right\|_{H^{1}(\Omega)}^{2}+\inf (\lambda, \tilde{\lambda})\left\|\nabla \cdot \mathbf{e}_{J}\right\|_{L^{2}(\Omega)}^{2} \leq C\left(\frac{1}{\lambda^{J}}+\frac{1}{\tilde{\lambda}^{J}}\right)\left\|\nabla \cdot \mathbf{e}_{J}\right\|_{L^{2}(\Omega)} .
$$


It then follows that

$$
\left\|\nabla \cdot \mathbf{e}_{J}\right\|_{L^{2}(\Omega)} \leq C\left(\frac{1}{\lambda^{J+1}}+\frac{1}{\tilde{\lambda}^{J+1}}\right)
$$

and therefore

$$
\inf (\mu, \tilde{\mu})\left\|\mathbf{e}_{J}\right\|_{H^{1}(\Omega)}^{2} \leq C\left(\frac{1}{\lambda^{2 J+1}}+\frac{1}{\tilde{\lambda}^{2 J+1}}\right)
$$

as desired. This completes the proof.

It is appropriate to point out that the background solution $\mathbf{U}$ in the absence of any elastic anomaly can be approximated to the first-order for large $\lambda$ by $\mathbf{U}_{0}$ defined as the solution to

$$
\left\{\begin{array}{l}
\left(\mu \Delta+\omega^{2} \rho\right) \mathbf{U}_{0}+\nabla q_{0}=0 \quad \text { in } \Omega, \\
\nabla \cdot \mathbf{U}_{0}=0 \text { in } \Omega, \\
\mathbf{U}_{0}=\mathbf{g} \text { on } \partial \Omega, \\
\int_{\Omega} q_{0}=0 .
\end{array}\right.
$$

If $\mathbf{U}$ is a shear wave (i.e. divergence-free) then $q_{0}=0$, as it can be easily checked.

4. Modified Stokes system. We now seek to represent the solution to (3.4) in terms of layer potentials. For that purpose we first consider the following modified Stokes system:

$$
\left\{\begin{array}{l}
\left(\Delta+\kappa^{2}\right) \mathbf{v}-\nabla q=0 \\
\nabla \cdot \mathbf{v}=0
\end{array}\right.
$$

where

$$
\kappa:=\omega \sqrt{\frac{\rho}{\mu}} .
$$

If $\kappa=0$, then (4.1) becomes the standard Stokes system and we may view (4.1) as a compact perturbation of that system.

4.1. Layer potentials. Let $\partial_{j}$ denote $\partial / \partial x_{j}$. Fundamental tensors $\boldsymbol{\Gamma}^{\kappa}=\left(\Gamma_{i j}^{\kappa}\right)_{i, j=1}^{3}$ and $\mathbf{F}=\left(F_{i}\right)_{i=1}^{3}$ to $(4.1)$ in three dimensions are given by

$$
\left\{\begin{array}{l}
\Gamma_{i j}^{\kappa}(x)=-\frac{\delta_{i j}}{4 \pi} \frac{e^{\sqrt{-1} \kappa|x|}}{|x|}-\frac{1}{4 \pi \kappa^{2}} \partial_{i} \partial_{j} \frac{e^{\sqrt{-1} \kappa|x|}-1}{|x|} \\
F_{i}(x)=-\frac{1}{4 \pi} \frac{x_{i}}{|x|^{3}} .
\end{array}\right.
$$

In fact, since $\frac{e^{\sqrt{-1} \kappa|x|}}{4 \pi|x|}$ is a fundamental solution to the Helmholtz operator $\Delta+\kappa^{2}$ and

$$
\partial_{i} \partial_{j} \frac{e^{\sqrt{-1}} \kappa|x|}{|x|}=\sum_{k=1}^{\infty} \frac{(\sqrt{-1} \kappa)^{k+1}}{(k+1) !}\left[k(k-2)|x|^{k-4} x_{i} x_{j}+k \delta_{i j}|x|^{k-2}\right],
$$

we have

$$
\left\{\begin{array}{l}
\left(\Delta+\kappa^{2}\right) \Gamma_{i j}^{\kappa}-\partial_{j} F_{i}=\delta_{i j} \delta(x), \quad \text { in } \mathbb{R}^{3}, \\
\partial_{i} \Gamma_{i j}^{\kappa}=0,
\end{array}\right.
$$


in the sense of distributions. Note that we used the Einstein convention for the summation notation omitting the summation sign for the indices appearing twice. We will continue using this convention throughout this paper. Moreover, we have from (4.4) that

$$
\Gamma_{i j}^{\kappa}(x)=\Gamma_{i j}^{0}(x)-\frac{\delta_{i j} \kappa \sqrt{-1}}{6 \pi}+O\left(\kappa^{2}\right),
$$

uniformly in $x$ as long as $|x|$ is bounded, where

$$
\Gamma_{i j}^{0}(x)=-\frac{1}{8 \pi}\left(\frac{\delta_{i j}}{|x|}+\frac{x_{i} x_{j}}{|x|^{3}}\right) .
$$

It is known (see [27] for example) that $\boldsymbol{\Gamma}^{0}=\left(\Gamma_{i j}^{0}\right)_{i, j=1}^{3}$ and $\mathbf{F}$ are the fundamental tensors for the standard Stokes system.

Let $D$ be a bounded $\mathcal{C}^{2}$-domain in $\mathbb{R}^{3}$ and introduce the single and double layer potentials on $\partial D$. For $i=1,2,3, \varphi=\left(\varphi_{1}, \varphi_{2}, \varphi_{3}\right) \in L^{2}(\partial D)^{3}$, and for $x \in \mathbb{R}^{3} \backslash \partial D$, let

$$
\left\{\begin{array}{l}
\mathbf{S}_{D}^{\kappa}[\varphi]_{i}(x):=\int_{\partial D} \Gamma_{i j}^{\kappa}(x-y) \varphi_{j}(y) d \sigma(y) \\
\mathcal{Q}_{D}[\varphi](x):=\int_{\partial D} F_{j}(x-y) \varphi_{j}(y) d \sigma(y)
\end{array}\right.
$$

and

$$
\left\{\begin{array}{l}
\mathbf{D}_{D}^{\kappa}[\varphi]_{i}(x):=\int_{\partial D}\left(\frac{\partial \Gamma_{i j}^{\kappa}}{\partial \mathbf{N}(y)}(x-y)+F_{i}(x-y) N_{j}(y)\right) \varphi_{j}(y) d \sigma(y), \\
\mathcal{V}_{D}[\varphi](x):=-2 \int_{\partial D} \frac{\partial}{\partial x_{l}} F_{j}(x-y) \varphi_{j}(y) N_{l}(y) d \sigma(y) .
\end{array}\right.
$$

Here $\mathbf{S}_{D}^{\kappa}[\varphi]_{i}$ and $\mathbf{D}_{D}^{\kappa}[\varphi]_{i}$ denote the $i$-th component of the vector-valued functions $\mathbf{S}_{D}^{\kappa}[\varphi]$ and $\mathbf{D}_{D}^{\kappa}[\varphi]$. Note that

$$
\frac{\partial \Gamma_{i j}^{\kappa}}{\partial \mathbf{N}(y)}(x-y)=\left(\frac{\partial \Gamma_{i j}^{\kappa}(x-y)}{\partial y_{l}}+\frac{\partial \Gamma_{i l}^{\kappa}(x-y)}{\partial y_{j}}\right) N_{l}(y) .
$$

Then $\left(\mathbf{S}_{D}^{\kappa}[\varphi], \mathcal{Q}_{D}[\varphi]\right)$ and $\left(\mathbf{D}_{D}^{\kappa}[\varphi], \mathcal{V}_{D}[\varphi]\right)$ satisfy $(4.1)$.

For convenience we introduce another notation for the conormal derivative:

$$
\left.\frac{\partial \mathbf{v}}{\partial n}\right|_{ \pm}:=\left.\frac{\partial \mathbf{v}}{\partial \mathbf{N}}\right|_{ \pm}-\left.q\right|_{ \pm} \mathbf{N} \quad \text { on } \partial D
$$

for a pair $(\mathbf{v}, q)$. Then, for any pairs $(\mathbf{u}, p)$ and $(\mathbf{v}, q)$ satisfying $\nabla \cdot \mathbf{u}=0$ and $\nabla \cdot \mathbf{v}=0$, the following Green's formulae hold (see [27]):

$$
\int_{\partial D} \mathbf{u} \cdot \frac{\partial \mathbf{v}}{\partial n} d \sigma=\int_{D} \frac{1}{2} \sum_{i, j=1}^{3}\left(\frac{\partial \mathbf{u}_{i}}{\partial x_{j}}+\frac{\partial \mathbf{u}_{j}}{\partial x_{i}}\right)\left(\frac{\partial \mathbf{v}_{i}}{\partial x_{j}}+\frac{\partial \mathbf{v}_{j}}{\partial x_{i}}\right)+\mathbf{u} \cdot(\triangle \mathbf{v}-\nabla q) d x
$$

Using (4.5) and (4.11), one can obtain a representation formula for any solution $(\mathbf{v}, q)$ to (4.3):

$$
\left\{\begin{array}{l}
\mathbf{v}(x)=-\mathbf{S}_{D}^{\kappa}\left[\left.\frac{\partial \mathbf{v}}{\partial n}\right|_{-}\right](x)+\mathbf{D}_{D}^{\kappa}[\mathbf{v}](x), \\
q(x)=-\mathcal{Q}_{D}\left[\left.\frac{\partial \mathbf{v}}{\partial n}\right|_{-}\right](x)+\mathcal{V}_{D}[\mathbf{v}](x),
\end{array} \quad x \in D\right.
$$


For $\varphi \in L^{2}(\partial D)^{3}$, the following trace relations for $\mathbf{D}_{D}^{\kappa}$ and the conormal derivative of $\mathbf{S}_{D}^{\kappa}$ hold:

$$
\begin{aligned}
\left.\mathbf{D}_{D}^{\kappa}[\varphi]\right|_{ \pm} & =\left(\mp \frac{1}{2} I+\mathbf{K}_{D}^{\kappa}\right)[\varphi] \quad \text { a.e. on } \partial D \\
\left.\frac{\partial}{\partial n} \mathbf{S}_{D}^{\kappa}[\varphi]\right|_{ \pm} & =\left( \pm \frac{1}{2} I+\left(\mathbf{K}_{D}^{\kappa}\right)^{*}\right)[\varphi] \quad \text { a.e. on } \partial D
\end{aligned}
$$

where $\mathbf{K}_{D}^{\kappa}$ is defined by

$$
\mathbf{K}_{D}^{\kappa}[\varphi]_{i}(x):=\text { p.v. } \int_{\partial D} \frac{\partial \Gamma_{i j}^{\kappa}}{\partial \mathbf{N}(y)}(x-y) \varphi_{j}(y) d \sigma(y)+\text { p.v. } \int_{\partial D} F_{i}(x-y) \varphi_{j}(y) N_{j}(y) d \sigma(y),
$$

for almost all $x \in \partial D$, and $\left(\mathbf{K}_{D}^{\kappa}\right)^{*}$ is the adjoint operator of $\mathbf{K}_{D}^{\kappa}$ on $L^{2}(\partial D)$, that is,

$$
\left(\mathbf{K}_{D}^{\kappa}\right)^{*}[\varphi]_{i}(x):=\text { p.v. } \int_{\partial D} \frac{\partial \Gamma_{i j}^{\kappa}}{\partial \mathbf{N}(x)}(x-y) \varphi_{j}(y) d \sigma(y)-\text { p.v. } \int_{\partial D} F_{i}(x-y) \varphi_{j}(y) N_{j}(x) d \sigma(y) .
$$

In fact, the formulas (4.13) and (4.14) were proved in [27] when $\kappa=0$. Since $\mathbf{D}_{D}^{\kappa}-\mathbf{D}_{D}^{0}$ and $\mathbf{S}_{D}^{\kappa}-\mathbf{S}_{D}^{0}$ are smoothing operators according to (4.6), we obtain (4.13) and (4.14) when $\kappa \neq 0$. It would be of use to readers to note that by putting together the two integrals in (4.15), we have

$$
\mathbf{K}_{D}^{0}[\varphi]_{i}(x):=-\frac{3}{4 \pi} \int_{\partial D} \frac{\langle x-y, \mathbf{N}(y)\rangle\left(x_{i}-y_{i}\right)\left(x_{j}-y_{j}\right)}{|x-y|^{5}} \varphi_{j}(y) d \sigma(y) .
$$

If $\partial D$ is $\mathcal{C}^{2}$ as we assume it to be, then

$$
|\langle x-y, \mathbf{N}(y)\rangle| \leq C|x-y|^{2},
$$

and hence $\mathbf{K}_{D}^{0}$ is a compact operator on $L^{2}(\partial D)^{3}$.

Formulae (4.13) and (4.14) show, in particular, that the double and single layer potentials obey the following jump relations on $\partial D$ :

$$
\begin{aligned}
\left.\mathbf{D}_{D}^{\kappa}[\varphi]\right|_{+}-\left.\mathbf{D}_{D}^{\kappa}[\varphi]\right|_{-} & =-\varphi \quad \text { a.e. on } \partial D \\
\left.\frac{\partial}{\partial n} \mathbf{S}_{D}^{\kappa}[\varphi]\right|_{+}-\left.\frac{\partial}{\partial n} \mathbf{S}_{D}^{\kappa}[\varphi]\right|_{-} & =\varphi \quad \text { a.e. on } \partial D
\end{aligned}
$$

On the other hand, the conormal derivative of the double layer potentials does not have a jump. In fact, if $\varphi \in H^{1}(\partial D)^{3}$, then

$$
\begin{aligned}
\left.\frac{\partial}{\partial n}\left(\mathbf{D}_{D}^{\kappa}[\varphi]\right)_{i}\right|_{+}(x) & =\left.\frac{\partial}{\partial n}\left(\mathbf{D}_{D}^{\kappa}[\varphi]\right)_{i}\right|_{-}(x) \\
& =\text { p.v. } \int_{\partial D} \frac{\partial^{2} \Gamma_{i j}^{\kappa}}{\partial \mathbf{N}(x) \partial \mathbf{N}(y)}(x-y) \varphi_{j}(y) d \sigma(y) \quad \text { a.e. on } \partial D .
\end{aligned}
$$

Lemma 4.1. Let $L_{0}^{2}(\partial D):=\left\{\mathbf{g} \in L^{2}(\partial D)^{3} \mid \int_{\partial D} \mathbf{g} \cdot \mathbf{N}=0\right\}$, and define $H_{0}^{1}(\partial D)$ likewise. Let $L:=\operatorname{ker}\left(\frac{1}{2} I+\mathbf{K}_{D}^{0}\right)^{\perp}$ in $L^{2}(\partial D)^{3}$. Then the following holds.

(i) $\mathbf{S}_{D}^{0}: L_{0}^{2}(\partial D) \rightarrow H_{0}^{1}(\partial D)$ is invertible.

(ii) $\frac{1}{2} I+\mathbf{K}_{D}^{0}: L \rightarrow L_{0}^{2}(\partial D)$ is invertible and so is $\frac{1}{2} I+\left(\mathbf{K}_{D}^{0}\right)^{*}: L_{0}^{2}(\partial D) \rightarrow L$.

(iii) $\lambda I+\mathbf{K}_{D}^{0}$ and $\lambda I+\left(\mathbf{K}_{D}^{0}\right)^{*}$ are invertible on $L^{2}(\partial D)^{3}$ for $|\lambda|>\frac{1}{2}$. 
Proof. The assertion (ii) was proved in [27]. We also recall from [27] that

$$
\operatorname{ker} \mathbf{S}_{D}^{0}=\operatorname{ker}\left(\frac{1}{2} I+\left(\mathbf{K}_{D}^{0}\right)^{*}\right)=\langle\mathbf{N}\rangle
$$

where $\mathbf{N}$ is the outward normal to $\partial D$. It implies that $L=L_{0}^{2}(\partial D)$.

To prove (i), we first note that $\mathbf{S}_{D}^{0}: L_{0}^{2}(\partial D) \rightarrow H_{0}^{1}(\partial D)$ is one-to-one because of (4.22). To prove that it is onto, let $\mathbf{g} \in H_{0}^{1}(\partial D)$ and let $\mathbf{v}$ be the solution to the exterior problem for the Stokes system, i.e.,

$$
\begin{cases}\Delta \mathbf{v}-\nabla q=0 & \text { in } \mathbb{R}^{3} \backslash \bar{D} \\ \nabla \cdot \mathbf{v}=0 & \text { in } \mathbb{R}^{3} \backslash \bar{D} \\ \mathbf{v}=\mathbf{g} & \text { on } \partial D \\ \mathbf{v}(x)=O\left(|x|^{-2}\right) & \text { as }|x| \rightarrow \infty .\end{cases}
$$

Let $\phi \in L^{2}(\partial D)^{3}$ satisfy $\left(\frac{1}{2} I+\mathbf{K}_{D}^{0}\right)[\phi]=0$ on $\partial D$. Then, because of (4.18), we have $\mathbf{K}_{D}^{0} \phi \in H^{1}(\partial D)^{3}$, and hence $\phi \in H^{1}(\partial D)^{3}$. Moreover, by (4.13), we have $\mathbf{D}_{D}^{0}[\phi]=0$ in $D$, and the corresponding pressure $q=c$ in $D$ for some constant $c$. It thus follows from (4.22) and (4.21) that

$$
\left.\frac{\partial \mathbf{D}_{D}^{0}[\phi]}{\partial n}\right|_{+}=\left.\frac{\partial \mathbf{D}_{D}^{0}[\phi]}{\partial n}\right|_{-}=\left.\frac{\partial \mathbf{D}_{D}^{0}[\phi]}{\partial \mathbf{N}}\right|_{-}-\left.q\right|_{-} \mathbf{N}=-c \mathbf{N}
$$

Applying the Green's formula, we have

$$
\int_{\partial D} \frac{\partial \mathbf{v}}{\partial n} \cdot \phi=-\int_{\partial D} \frac{\partial \mathbf{v}}{\partial n} \cdot\left(-\frac{1}{2} I+\mathbf{K}_{D}^{0}\right) \phi=-\left.\int_{\partial D} \mathbf{v} \cdot \frac{\partial \mathbf{D}_{D}^{0}[\phi]}{\partial n}\right|_{+}=0
$$

Thus $\frac{\partial \mathbf{v}}{\partial n} \in L$. Let

$$
\psi:=\left(\frac{1}{2} I+\left(\mathbf{K}_{D}^{0}\right)^{*}\right)^{-1}\left[\frac{\partial \mathbf{v}}{\partial n}\right] \text { on } \partial D
$$

Then, by (4.14), we get

$$
\left.\frac{\partial\left(\mathbf{S}_{D}^{0}[\psi]\right)}{\partial n}\right|_{+}=\left.\frac{\partial \mathbf{v}}{\partial n}\right|_{+}
$$

and hence $\mathbf{S}_{D}^{0}[\psi]=\mathbf{v}$ in $\mathbb{R}^{3} \backslash D$. In particular, $\mathbf{S}_{D}^{0}[\psi]=\mathbf{g}$ and hence $\mathbf{S}_{D}^{0}: L_{0}^{2}(\partial D) \rightarrow$ $H_{0}^{1}(\partial D)$ is onto. Thus, we obtain (i). 
To prove (iii), suppose that $\left(\lambda I+\left(\mathbf{K}_{D}^{0}\right)^{*}\right)[\phi]=0$ on $\partial D$. By the Green's formula (4.11), we have

$$
\begin{aligned}
& \frac{1}{2} \int_{D} \sum_{i, k=1}^{3}\left(\frac{\partial \mathbf{S}_{D}^{0}[\phi]_{i}}{\partial x_{k}}+\frac{\partial \mathbf{S}_{D}^{0}[\phi]_{k}}{\partial x_{i}}\right)^{2} d x \\
& =\int_{\partial D}\left(-\frac{1}{2} I+\left(\mathbf{K}_{D}^{0}\right)^{*}\right)[\phi] \cdot \mathbf{S}_{D}^{0}[\phi] d \sigma \\
& =\frac{\lambda+\frac{1}{2}}{\lambda-\frac{1}{2}} \int_{\partial D}\left(\frac{1}{2} I+\left(\mathbf{K}_{D}^{0}\right)^{*}\right)[\phi] \cdot \mathbf{S}_{D}^{0}[\phi] d \sigma \\
& =-\frac{1}{2} \frac{\lambda+\frac{1}{2}}{\lambda-\frac{1}{2}} \int_{\mathbb{R}^{3} \backslash \bar{D}} \sum_{i, k=1}^{3}\left(\frac{\partial \mathbf{S}_{D}^{0}[\phi]_{i}}{\partial x_{k}}+\frac{\partial \mathbf{S}_{D}^{0}[\phi]_{k}}{\partial x_{i}}\right)^{2} d x .
\end{aligned}
$$

Since $\frac{\lambda+\frac{1}{2}}{\lambda-\frac{1}{2}}>0$, we have

$$
\frac{\partial \mathbf{S}_{D}^{0}[\phi]_{i}}{\partial x_{k}}+\frac{\partial \mathbf{S}_{D}^{0}[\phi]_{k}}{\partial x_{i}}=0 \quad \text { in } \mathbb{R}^{3} \backslash \partial D, \quad i, j=1,2,3
$$

which implies that $\mathbf{S}_{D}^{0}[\phi]=C$ in $\mathbb{R}^{3} \backslash \bar{D}$ for some constant $C$. On the other hand, $\mathbf{S}_{D}^{0}[\phi]$ vanishes at infinity, and hence it vanishes in $\mathbb{R}^{3}$. Therefore, we have

$$
\phi=\frac{1}{\lambda-\frac{1}{2}}\left(\left(\lambda I+\left(\mathbf{K}_{D}^{0}\right)^{*}\right) \phi-\left(\frac{1}{2} I+\left(\mathbf{K}_{D}^{0}\right)^{*}\right) \phi\right)=0 .
$$

Thus $\left(\lambda I+\left(\mathbf{K}_{D}^{0}\right)^{*}\right)$ is injective on $L^{2}(\partial D)^{3}$. Since $\left(\mathbf{K}_{D}^{0}\right)^{*}$ is compact on $L^{2}(\partial D)^{3}$ by (4.18), we have (iii) by the Fredholm alternative. This completes the proof.

4.2. Transmission problems. We now consider the transmission problem (3.4). If we put $\tilde{\kappa}:=\omega \sqrt{\frac{\tilde{\rho}}{\tilde{\mu}}}$ and

$$
q_{0}:= \begin{cases}-\frac{1}{\mu} p_{0} & \text { in } \Omega \backslash \bar{D} \\ -\frac{1}{\tilde{\mu}} p_{0} & \text { in } D,\end{cases}
$$

then (3.4) takes the form

$$
\left\{\begin{array}{l}
\left(\Delta+\kappa^{2}\right) \mathbf{u}_{0}-\nabla q_{0}=0 \quad \text { in } \Omega \backslash \bar{D} \\
\left(\Delta+\tilde{\kappa}^{2}\right) \mathbf{u}_{0}-\nabla q_{0}=0 \quad \text { in } D \\
\left.\mathbf{u}_{0}\right|_{+}-\left.\mathbf{u}_{0}\right|_{-}=0 \quad \text { on } \partial D \\
\left.\mu \frac{\partial \mathbf{u}_{0}}{\partial n}\right|_{+}-\left.\tilde{\mu} \frac{\partial \mathbf{u}_{0}}{\partial n}\right|_{-}=0 \quad \text { on } \partial D \\
\nabla \cdot \mathbf{u}_{0}=0 \quad \text { in } \Omega \\
\mathbf{u}_{0}=\mathbf{g} \text { on } \partial \Omega \\
\int_{\Omega} q_{0}=0, \quad \int_{\partial \Omega} \mathbf{g} \cdot \mathbf{N}=0
\end{array}\right.
$$


We look for the solution to (4.28) in the form of

$$
\mathbf{u}_{0}= \begin{cases}\mathbf{S}_{D}^{\tilde{\kappa}}[\phi] & \text { in } D, \\ \mathbf{S}_{D}^{\kappa}[\psi]+\mathbf{D}_{\Omega}^{\kappa}[\theta] & \text { in } \Omega \backslash \bar{D},\end{cases}
$$

for some triplet $(\phi, \psi, \theta) \in L^{2}(\partial D)^{3} \times L^{2}(\partial D)^{3} \times L^{2}(\partial \Omega)^{3}$. In view of the transmission conditions on $\partial D$ and the boundary condition on $\partial \Omega$ in $(4.28),(\phi, \psi, \theta)$ should satisfy the following system of integral equations:

$$
\begin{cases}\mathbf{S}_{D}^{\tilde{\kappa}}[\phi]-\mathbf{S}_{D}^{\kappa}[\psi]-\mathbf{D}_{\Omega}^{\kappa}[\theta]=0 & \text { on } \partial D \\ \tilde{\mu}\left(-\frac{1}{2} I+\left(\mathbf{K}_{D}^{\tilde{\kappa}}\right)^{*}\right)[\phi]-\mu\left(\frac{1}{2} I+\left(\mathbf{K}_{D}^{\kappa}\right)^{*}\right)[\psi]-\mu \frac{\partial}{\partial n} \mathbf{D}_{\Omega}^{\kappa}[\theta]=0 & \text { on } \partial D \\ \mathbf{S}_{D}^{\kappa}[\psi]+\left(\frac{1}{2} I+\mathbf{K}_{\Omega}^{\kappa}\right)[\theta]=\mathbf{g} & \text { on } \partial \Omega\end{cases}
$$

or equivalently

$$
\left(\begin{array}{ccc}
\mathbf{S}_{D}^{\tilde{\kappa}} & -\mathbf{S}_{D}^{\kappa} & -\mathbf{D}_{\Omega}^{\kappa} \\
\tilde{\mu}\left(-\frac{1}{2} I+\left(\mathbf{K}_{D}^{\tilde{\kappa}}\right)^{*}\right) & -\mu\left(\frac{1}{2} I+\left(\mathbf{K}_{D}^{\kappa}\right)^{*}\right) & -\mu \frac{\partial}{\partial n} \mathbf{D}_{\Omega}^{\kappa} \\
0 & \mathbf{S}_{D}^{\kappa} & \frac{1}{2} I+\mathbf{K}_{\Omega}^{\kappa}
\end{array}\right)\left(\begin{array}{c}
\phi \\
\psi \\
\theta
\end{array}\right)=\left(\begin{array}{l}
0 \\
0 \\
\mathbf{g}
\end{array}\right)
$$

Denote the operator on the left-hand side of (4.30) by $A_{\kappa}$. Then $A_{\kappa}$ maps $L^{2}(\partial D)^{3} \times$ $L^{2}(\partial D)^{3} \times L^{2}(\partial \Omega)^{3}$ into $H_{0}^{1}(\partial D) \times L^{2}(\partial D)^{3} \times L_{0}^{2}(\partial \Omega)$.

We now investigate the solvability of the equation (4.30). Because of (4.6), $A_{\kappa}$ is a compact perturbation of $A_{0}$, which is again a compact perturbation of

$$
\left(\begin{array}{ccc}
\mathbf{S}_{D}^{0} & -\mathbf{S}_{D}^{0} & 0 \\
\tilde{\mu}\left(-\frac{1}{2} I+\left(\mathbf{K}_{D}^{0}\right)^{*}\right) & -\mu\left(\frac{1}{2} I+\left(\mathbf{K}_{D}^{0}\right)^{*}\right) & 0 \\
0 & 0 & \frac{1}{2} I+\mathbf{K}_{\Omega}^{0}
\end{array}\right)
$$

noting that the operators $\mathbf{D}_{\Omega}^{\kappa}$ and $\frac{\partial}{\partial n} \mathbf{D}_{\Omega}^{\kappa}$ in the third column of $A_{\kappa}$ are compact as operators from $L^{2}(\partial D)^{3}$ into $H_{0}^{1}(\partial D)$ and $L^{2}(\partial D)^{3}$ since $\partial D$ and $\partial \Omega$ do not intersect. Define

$$
S:=\left\{(\phi, \psi) \in L^{2}(\partial D)^{3} \times L^{2}(\partial D)^{3}: \phi-\psi \in L_{0}^{2}(\partial D)\right\} .
$$

Denote $\operatorname{ker}\left(\frac{1}{2} I+\mathbf{K}_{\Omega}^{\kappa}\right)^{\perp}$ by $L_{\kappa}$. Then the following holds.

Lemma 4.2. The operator $\mathbf{A}_{0}: S \times L_{0} \rightarrow H_{0}^{1}(\partial D) \times L^{2}(\partial D)^{3} \times L_{0}^{2}(\partial \Omega)$ is invertible. So is $A_{\kappa}: S \times L_{\kappa}$ into $H_{0}^{1}(\partial D) \times L^{2}(\partial D)^{3} \times L_{0}^{2}(\partial \Omega)$, provided that $\kappa^{2}$ is not a Dirichlet eigenvalue of the Stokes system on either $D$ or $\Omega$.

Proof. Using Lemma 4.1, one can easily show that the operator in (4.31) is invertible. Since $\mathbf{A}_{0}$ is its compact perturbation, it suffices to show that $\mathbf{A}_{0}$ is injective according to the Fredholm alternative.

Suppose that there exists $\left(\phi_{0}, \psi_{0}, \theta_{0}\right) \in S \times L_{0}$ such that

$$
\mathbf{A}_{0}\left[\begin{array}{l}
\phi_{0} \\
\psi_{0} \\
\theta_{0}
\end{array}\right]=0 .
$$


Then the function $\mathbf{v}$, defined by

$$
\mathbf{v}(x):= \begin{cases}\mathbf{S}_{D}^{0}\left[\phi_{0}\right](x), & x \in D, \\ \mathbf{S}_{D}^{0}\left[\psi_{0}\right](x)+\mathbf{D}_{\Omega}^{0}\left[\theta_{0}\right](x), & x \in \Omega \backslash \bar{D},\end{cases}
$$

is a solution to the problem (4.28) with $\kappa=\tilde{\kappa}=0$ and $\mathbf{g}=0$. Since the solution to (4.28) with $\kappa=\tilde{\kappa}=0$ is unique, we have

$$
\begin{aligned}
\mathbf{S}_{D}^{0}\left[\phi_{0}\right] & =0 & & \text { in } D, \\
\mathbf{S}_{D}^{0}\left[\psi_{0}\right]+\mathbf{D}_{\Omega}^{0}\left[\theta_{0}\right] & =0 & & \text { in } \Omega \backslash \bar{D} .
\end{aligned}
$$

Then (4.34) shows that $\mathbf{S}_{D}^{0}\left[\psi_{0}\right]$ can be extended to $\Omega$ as a solution to (4.1). Then by (4.20) we obtain $\psi_{0}=c \mathbf{N}$ for some constant $c$, and $\mathbf{D}_{\Omega}^{0}\left[\theta_{0}\right]=0$ in $\Omega$. By (4.13) and part (ii) in Lemma 4.1 , we have $\theta_{0}=0$. On the other hand,

$$
\phi_{0}=\left.\frac{\partial \mathbf{S}_{D}^{0}\left[\phi_{0}\right]}{\partial n}\right|_{+}-\left.\frac{\partial \mathbf{S}_{D}^{0}\left[\phi_{0}\right]}{\partial n}\right|_{-}=\left.\frac{\partial \mathbf{S}_{D}^{0}\left[\phi_{0}\right]}{\partial n}\right|_{+}-\left.\frac{\mu}{\tilde{\mu}} \frac{\partial \mathbf{S}_{D}^{0}\left[\psi_{0}\right]}{\partial n}\right|_{+}=0
$$

and hence $\psi_{0}=0$. Hence $\mathbf{A}_{0}$ is invertible.

Since the operator in (4.30) is a compact perturbation of $\mathbf{A}_{0}$, we can show that it is invertible in exactly the same manner under the assumption that $\kappa^{2}$ is not a Dirichlet eigenvalue of the Stokes system in either $D$ or $\Omega$. This completes the proof.

Thus we obtain the following theorem.

Theorem 4.3. Let $(\phi, \psi, \theta) \in S \times L_{\kappa}$ be the unique solution to (4.30). Then the solution $\mathbf{u}_{0}$ to $(4.27)$ is represented by $(4.29)$.

Consider the following boundary-value problem for the modified Stokes system in the absence of the elastic anomaly:

$$
\begin{cases}\left(\Delta+\kappa^{2}\right) \mathbf{v}+\nabla q=0 & \text { in } \Omega \\ \nabla \cdot \mathbf{v}=0 & \text { in } \Omega \\ \mathbf{v}=\mathbf{g} & \text { on } \partial \Omega \\ \int_{\Omega} q=0, & \end{cases}
$$

under the compatibility condition $\int_{\partial \Omega} \mathbf{g} \cdot \mathbf{N}=0$. Let

$$
\theta_{0}=\left(\frac{1}{2} I+\mathbf{K}_{\Omega}^{\kappa}\right)^{-1}[\mathbf{g}] \quad \text { on } \partial \Omega .
$$

Then the solution $\mathbf{U}_{0}$ to (4.36) is given by

$$
\mathbf{U}_{0}(x)=\mathbf{D}_{\Omega}^{\kappa}\left[\theta_{0}\right](x)=\mathbf{D}_{\Omega}^{\kappa}\left(\frac{1}{2} I+\mathbf{K}_{\Omega}^{\kappa}\right)^{-1}[\mathbf{g}](x), \quad x \in \Omega .
$$

By (4.29), we have

$$
\begin{aligned}
\mathbf{D}_{\Omega}^{\kappa}\left(\frac{1}{2} I+\mathbf{K}_{\Omega}^{\kappa}\right)^{-1}\left[\mathbf{g}-\left.\mathbf{S}_{D}^{\kappa}[\psi]\right|_{\partial \Omega}\right] & =\mathbf{D}_{\Omega}^{\kappa}\left(\frac{1}{2} I+\mathbf{K}_{\Omega}^{\kappa}\right)^{-1}\left[\left.\mathbf{D}_{\Omega}^{\kappa}[\theta]\right|_{-}\right] \\
& =\mathbf{D}_{\Omega}^{\kappa}[\theta]
\end{aligned}
$$

and hence we obtain

$$
\mathbf{u}_{0}(x)=\mathbf{U}_{0}(x)+\mathbf{S}_{D}^{\kappa}[\psi](x)-\mathbf{D}_{\Omega}^{\kappa}\left(\frac{1}{2}+\mathbf{K}_{\Omega}^{\kappa}\right)^{-1}\left[\left.\mathbf{S}_{D}^{\kappa}[\psi]\right|_{\partial \Omega}\right](x), \quad x \in \Omega \backslash \bar{D} .
$$


Let $\mathbf{G}^{\kappa}=\left(G_{i j}^{\kappa}\right)_{i, j=1}^{3}$ be the Green's function to the Dirichlet problem for the operator in (4.1) in $\Omega$, i.e., for $y \in \Omega$,

$$
\left\{\begin{array}{l}
\left(\Delta_{x}+\kappa^{2}\right) G_{i j}^{\kappa}(x, y)-\frac{\partial F_{i}(x-y)}{\partial x_{j}}=\delta_{i j} \delta_{y}(x) \quad \text { in } \Omega \\
\sum_{j=1}^{3} \frac{\partial}{\partial x_{j}} G_{i j}^{\kappa}(x, y)=0 \quad \text { in } \Omega \\
G_{i j}^{\kappa}(x, y)=0 \quad \text { on } \partial \Omega
\end{array}\right.
$$

Define for $\mathbf{f} \in L_{0}^{2}(\partial D)$

$$
\mathbf{G}_{D}^{\kappa}[\mathbf{f}](x):=\int_{\partial D} \mathbf{G}^{\kappa}(x, y) \mathbf{f}(y) d \sigma, \quad x \in \Omega .
$$

Then the following identity holds:

$$
\mathbf{G}_{D}^{\kappa}[\mathbf{f}](x)=\mathbf{S}_{D}^{\kappa}[\mathbf{f}](x)-\mathbf{D}_{\Omega}^{\kappa}\left(\frac{1}{2}+\mathbf{K}_{\Omega}^{\kappa}\right)^{-1} \mathbf{S}_{D}^{\kappa}[\mathbf{f}](x), \quad x \in \Omega .
$$

In fact, by the definition of the Green's function, we have

$$
\mathbf{G}^{\kappa}(x, y)=\boldsymbol{\Gamma}^{\kappa}(x, y)-\mathbf{D}_{\Omega}^{\kappa}\left(\frac{1}{2}+\mathbf{K}_{\Omega}^{\kappa}\right)^{-1}\left[\boldsymbol{\Gamma}^{\kappa}(\cdot, y)\right](x), \quad x \in \Omega .
$$

From (4.39), we immediately obtain the following theorem.

Theorem 4.4. Let $(\phi, \psi, \theta) \in S \times L_{\kappa}$ be the unique solution to (4.30). Then

$$
\mathbf{u}_{0}(x)=\mathbf{U}_{0}(x)+\mathbf{G}_{D}^{\kappa}[\psi](x), \quad x \in \Omega \backslash \bar{D} .
$$

5. Small volume asymptotic expansions. In this section we rigorously derive the leading-order term in the asymptotic expansion of $\mathbf{u}_{0}$ as the volume of the anomaly $D$ goes to zero. Our asymptotic formula yields a very effective algorithm for reconstructing the shape and the shear modulus of the anomaly from MRE measurements.

5.1. Formal derivation of small volume asymptotic expansions. Recall that $D=\epsilon B+z$ and $\operatorname{dist}(D, \partial \Omega)>c_{0}$. We first give a formal derivation of the leading-order term in the asymptotic expansion of $\mathbf{u}_{0}$ as $\epsilon \rightarrow 0$. The purpose of this formal derivation is to see what kinds of formulas are expected to hold. This formula will be justified rigorously in a later section. Let $\left(\mathbf{U}_{0}, q_{0}\right)$ denote the background solution to the modified Stokes system (3.10), that is, the solution in the absence of the anomaly $D$.

To reveal the nature of the perturbations in $\mathbf{u}_{0}$, we introduce the local variables $\xi=(y-z) / \epsilon$ for $y \in \Omega$, and set $\hat{\mathbf{u}}_{0}(\xi)=\mathbf{u}_{0}(z+\epsilon \xi)$. We expect that $\mathbf{u}_{0}(y)$ will differ appreciably from $\mathbf{U}_{0}(y)$ for $y$ near $z$, but it will differ little from $\mathbf{U}_{0}(y)$ for $y$ far from $z$. Therefore, in the spirit of matched asymptotic expansions, we shall represent the field $\mathbf{u}_{0}$ (and $p_{0}$ ) by two different expansions, an inner expansion for $y$ near $z$, and an outer expansion for $y$ far from $z$. The outer expansion must begin with $\mathbf{U}_{0}$ (respectively $q_{0}$ ), so we write:

$$
\left\{\begin{array}{l}
\mathbf{u}_{0}(y)=\mathbf{U}_{0}(y)+\epsilon^{\tau_{1}} \mathbf{U}_{1}(y)+\epsilon^{\tau_{2}} \mathbf{U}_{2}(y)+\ldots, \\
p_{0}(y)=q_{0}(y)+\epsilon^{\tau_{1}} q_{1}(y)+\epsilon^{\tau_{2}} q_{2}(y)+\ldots,
\end{array} \quad \text { for }|y-z| \gg O(\epsilon),\right.
$$


where $0<\tau_{1}<\tau_{2}<\ldots, \mathbf{U}_{1}, \mathbf{U}_{2}, \ldots$, and $q_{1}, q_{2}, \ldots$, are to be found. We write the inner expansion as

$$
\left\{\begin{array}{l}
\hat{\mathbf{u}}_{0}(\xi)=\mathbf{u}_{0}(z+\epsilon \xi)=\hat{\mathbf{v}}_{0}(\xi)+\epsilon \hat{\mathbf{v}}_{1}(\xi)+\epsilon^{2} \hat{\mathbf{v}}_{2}(\xi)+\ldots, \\
\hat{p}_{0}(\xi)=p_{0}(z+\epsilon \xi)=\hat{p}_{0}(\xi)+\epsilon \hat{p}_{1}(\xi)+\epsilon^{2} \hat{p}_{2}(\xi)+\ldots,
\end{array} \quad \text { for }|\xi|=O(1),\right.
$$

where $\hat{\mathbf{v}}_{0}, \hat{\mathbf{v}}_{1}, \ldots$, are to be found. We assume that the functions $\hat{\mathbf{v}}_{j}, j=0,1, \ldots$, are defined not just in the domain obtained by stretching $\Omega$, but everywhere in $\mathbb{R}^{3}$.

Evidently, the functions $\hat{\mathbf{v}}_{i}$ are not defined uniquely, and the question now arises as to how to choose them. Thus, there is an arbitrariness in the choice of the coefficients of both the outer and the inner expansions. In order to determine the functions $\mathbf{U}_{i}(y), q_{i}(y)$ and $\hat{\mathbf{v}}_{i}(\xi), \hat{p}_{i}(\xi)$, we have to equate the inner and the outer expansions in some overlap domain within which the stretched variable $\xi$ is large and $y-z$ is small. In this domain the matching conditions are:

$$
\mathbf{U}_{0}(y)+\epsilon^{\tau_{1}} \mathbf{U}_{1}(y)+\epsilon^{\tau_{2}} \mathbf{U}_{2}(y)+\ldots \sim \hat{\mathbf{v}}_{0}(\xi)+\epsilon \hat{\mathbf{v}}_{1}(\xi)+\epsilon^{2} \hat{\mathbf{v}}_{2}(\xi)+\ldots
$$

and

$$
q_{0}(y)+\epsilon^{\tau_{1}} q_{1}(y)+\epsilon^{\tau_{2}} q_{2}(y)+\ldots \sim \hat{p}_{0}(\xi)+\epsilon \hat{p}_{1}(\xi)+\epsilon^{2} \hat{p}_{2}(\xi)+\ldots
$$

If we substitute the inner expansion into the transmission problem (3.4) and formally equate coefficients of $\epsilon^{-2}, \epsilon^{-1}$ we get $\hat{\mathbf{v}}_{0}(\xi)=\mathbf{U}_{0}(z)$, and

$$
\left\{\begin{array}{l}
\mu \Delta \hat{\mathbf{v}}_{1}+\nabla \hat{p}_{0}=0 \quad \text { in } \mathbb{R}^{3} \backslash \bar{B}, \\
\tilde{\mu} \Delta \hat{\mathbf{v}}_{1}+\nabla \hat{p}_{0}=0 \quad \text { in } B, \\
\left.\hat{\mathbf{v}}_{1}\right|_{-}-\left.\hat{\mathbf{v}}_{1}\right|_{+}=0 \quad \text { on } \partial B \\
\left.\left(\hat{p}_{0} \mathbf{N}+\tilde{\mu} \frac{\partial \hat{\mathbf{v}}_{1}}{\partial \mathbf{N}}\right)\right|_{-}-\left.\left(\hat{p}_{0} \mathbf{N}+\mu \frac{\partial \hat{\mathbf{v}}_{1}}{\partial \mathbf{N}}\right)\right|_{+}=0 \quad \text { on } \partial B, \\
\nabla \cdot \hat{\mathbf{v}}_{1}=0 \quad \text { in } \mathbb{R}^{3}, \\
\hat{\mathbf{v}}_{1}(\xi)-\nabla \mathbf{U}_{0}(z) \xi \rightarrow 0 \quad \text { as }|\xi| \rightarrow+\infty, \\
\hat{p}_{0}(\xi) \rightarrow 0 \quad \text { as }|\xi| \rightarrow+\infty .
\end{array}\right.
$$

Therefore, we arrive at the following (inner) asymptotic formula:

$$
\mathbf{u}_{0}(x) \approx \mathbf{U}_{0}(z)+\epsilon \hat{\mathbf{v}}_{1}\left(\frac{x-z}{\epsilon}\right) \text { for } x \text { near } z .
$$

Note that $\nabla_{\xi} \cdot\left(\nabla \mathbf{U}_{0}(z) \xi\right)=\nabla \cdot \mathbf{U}_{0}(z)=0$ in $\mathbb{R}^{3}$. Therefore, we can prove in exactly the same manner as in Section 4.2 that $\hat{\mathbf{v}}_{1}$ admits the following representation:

$$
\hat{\mathbf{v}}_{1}(\xi)=\left\{\begin{array}{l}
\nabla \mathbf{U}_{0}(z) \xi+\mathbf{S}_{B}^{0}[\hat{\psi}](\xi) \quad \text { in } \mathbb{R}^{3} \backslash \bar{B} \\
\mathbf{S}_{B}^{0}[\hat{\phi}](\xi) \text { in } B
\end{array}\right.
$$

where $(\hat{\phi}, \hat{\psi})$ is the unique solution to

$$
\left\{\begin{array}{l}
\mathbf{S}_{B}^{0}[\hat{\phi}]-\mathbf{S}_{B}^{0}[\hat{\psi}]=\nabla \mathbf{U}_{0}(z) \xi \quad \text { on } \partial B \\
\tilde{\mu}\left(-\frac{1}{2}+\left(\mathbf{K}_{B}^{0}\right)^{*}\right)[\hat{\phi}]-\mu\left(\frac{1}{2}+\left(\mathbf{K}_{B}^{0}\right)^{*}\right)[\hat{\psi}]=\mu \frac{\partial}{\partial n}\left(\nabla \mathbf{U}_{0}(z) \xi\right) \quad \text { on } \partial B .
\end{array}\right.
$$


We now derive the outer expansion. Let $\kappa=\omega \sqrt{\rho / \mu}$. One can see from (3.4) and (3.10) that $\left(\mathbf{u}_{0}-\mathbf{U}_{0}, p_{0}-q_{0}\right)$ satisfies

$$
\left\{\begin{array}{l}
\left(\Delta+\kappa^{2}\right)\left(\mathbf{u}_{0}-\mathbf{U}_{0}\right)+\frac{1}{\mu} \nabla\left(p_{0}-q_{0}\right)=0 \quad \text { in } \Omega \backslash \bar{D} \\
\left(\Delta+\kappa^{2}\right)\left(\mathbf{u}_{0}-\mathbf{U}_{0}\right)+\frac{1}{\mu} \nabla\left(p_{0}-q_{0}\right)=\left(\kappa^{2}-\tilde{\kappa}^{2}\right) \mathbf{u}_{0}+\left(\frac{1}{\mu}-\frac{1}{\tilde{\mu}}\right) \nabla p_{0} \quad \text { in } D \\
\left.\left(\mathbf{u}_{0}-\mathbf{U}_{0}\right)\right|_{+}-\left.\left(\mathbf{u}_{0}-\mathbf{U}_{0}\right)\right|_{-}=0 \quad \text { on } \partial D \\
\left.\frac{1}{\mu}\left(p_{0}-q_{0}\right)\right|_{+} \mathbf{N}+\left.\frac{\partial}{\partial \mathbf{N}}\left(\mathbf{u}_{0}-\mathbf{U}_{0}\right)\right|_{+} \\
\quad=\left.\frac{1}{\mu}\left(p_{0}-q_{0}\right)\right|_{-} \mathbf{N}+\left.\frac{\partial}{\partial \mathbf{N}}\left(\mathbf{u}_{0}-\mathbf{U}_{0}\right)\right|_{-}+\left.\frac{\tilde{\mu}-\mu}{\mu} \frac{\partial \mathbf{u}_{0}}{\partial \mathbf{N}}\right|_{-} \quad \text { on } \partial D \\
\nabla \cdot\left(\mathbf{u}_{0}-\mathbf{U}_{0}\right)=0 \quad \text { in } \Omega \\
\mathbf{u}_{0}-\mathbf{U}_{0}=0 \quad \text { on } \partial \Omega
\end{array}\right.
$$

Integrating the first equation in (5.5) against the Green's function $\mathbf{G}^{\kappa}(x, y)$ over $y \in \Omega \backslash \bar{D}$ and using the divergence theorem, we obtain the following representation formula for $x \in \Omega$ :

$$
\begin{aligned}
\mathbf{u}_{0}(x)= & \mathbf{U}_{0}(x)+\left.\left(\frac{\tilde{\mu}}{\mu}-1\right) \int_{\partial D} \mathbf{G}^{\kappa}(x, y) \frac{\partial \mathbf{u}_{0}}{\partial \mathbf{N}}\right|_{-}(y) d \sigma(y)+\left(\frac{1}{\mu}-\frac{1}{\tilde{\mu}}\right) \int_{D} \mathbf{G}^{\kappa}(x, y) \nabla p_{0}(y) d y \\
& +\omega^{2}\left(\frac{\rho}{\mu}-\frac{\tilde{\rho}}{\tilde{\mu}}\right) \int_{D} \mathbf{G}^{\kappa}(x, y) \mathbf{u}_{0}(y) d y .
\end{aligned}
$$

Since

$$
\left.\left(\frac{\tilde{\mu}}{\mu}-1\right) \int_{\partial D} \frac{\partial \mathbf{u}_{0}}{\partial \mathbf{N}}\right|_{-}(y) d \sigma(y)+\left(\frac{1}{\mu}-\frac{1}{\tilde{\mu}}\right) \int_{D} \nabla p_{0}(y) d y=-\left(\frac{\tilde{\mu}}{\mu}-1\right) \tilde{\kappa}^{2} \int_{D} \mathbf{u}_{0} d y,
$$

as can be seen by integration by parts, we obtain from the inner expansion that for $x$ far away from $z$,

$$
\begin{aligned}
\mathbf{u}_{0}(x) & \approx \mathbf{U}_{0}(x)+\epsilon^{3} \sum_{i, j, \ell=1}^{3} \mathbf{e}_{i} \partial_{\ell} G_{i j}^{\kappa}(x, z)\left[\left.\left(\frac{\tilde{\mu}}{\mu}-1\right) \int_{\partial B}\left(\frac{\partial \hat{\mathbf{v}}_{1}}{\partial \mathbf{N}}\right)_{j}\right|_{-}(\xi) \xi_{\ell} d \sigma(\xi)\right. \\
& \left.+\left(\frac{1}{\mu}-\frac{1}{\tilde{\mu}}\right) \int_{B} \partial_{j} \hat{p}_{0}(\xi) \xi_{\ell} d \xi\right]+\epsilon^{3}|B| \omega^{2} \frac{\rho-\tilde{\rho}}{\mu} \mathbf{G}^{\kappa}(x, z) \mathbf{U}_{0}(z),
\end{aligned}
$$

where $\left(\frac{\partial \hat{\mathbf{v}}_{1}}{\partial \mathbf{N}}\right)_{j}$ is the $j$-th component of $\frac{\partial \hat{\mathbf{v}}_{1}}{\partial \mathbf{N}}$, which we may simplify as follows:

$$
\begin{aligned}
\left(\mathbf{u}_{0}-\mathbf{U}_{0}\right)(x) & \approx \epsilon^{3}\left[\left(\frac{\tilde{\mu}}{\mu}-1\right) \sum_{i, j, \ell=1}^{3} \mathbf{e}_{i} \partial_{\ell} G_{i j}^{\kappa}(x, z) \int_{B} \partial_{j} \hat{\mathbf{v}}_{1 \ell}(\xi)+\partial_{\ell} \hat{\mathbf{v}}_{1 j}(\xi) d \xi\right. \\
& \left.+|B| \omega^{2} \frac{\rho-\tilde{\rho}}{\mu} \mathbf{G}^{\kappa}(x, z) \mathbf{U}_{0}(z)\right] .
\end{aligned}
$$

Here $\hat{\mathbf{v}}_{1 j}$ denotes the $j$-th component of $\hat{\mathbf{v}}_{1}$.

Formulae (5.2) and (5.6) are formally derived asymptotic inner and outer expansions. In the next section we represent these formulae using the notion of the viscous moment tensor. 
5.2. Viscous moment tensors. Let us now introduce the notion of a viscous moment tensor, which appears naturally in the representation of the outer expansion (5.6). For $\xi \in \mathbb{R}^{3}$, let $d(\xi):=\frac{1}{3} \sum_{k} \xi_{k} \mathbf{e}_{k}$ and let $\hat{\mathbf{v}}_{p q}, p, q=1,2,3$, be the solution to

$$
\left\{\begin{array}{l}
\mu \Delta \hat{\mathbf{v}}_{p q}+\nabla \hat{p}=0 \quad \text { in } \mathbb{R}^{3} \backslash \bar{B} \\
\tilde{\mu} \Delta \hat{\mathbf{v}}_{p q}+\nabla \hat{p}=0 \quad \text { in } B \\
\left.\hat{\mathbf{v}}_{p q}\right|_{-}-\left.\hat{\mathbf{v}}_{p q}\right|_{+}=0 \quad \text { on } \partial B \\
\left.\left.\left(\hat{p} \mathbf{N}+\tilde{\mu} \frac{\partial \hat{\mathbf{v}}_{p q}}{\partial \mathbf{N}}\right)\right|_{-}\left(\hat{p} \mathbf{N}+\mu \frac{\partial \hat{\mathbf{v}}_{p q}}{\partial \mathbf{N}}\right)\right|_{+}=0 \quad \text { on } \partial B \\
\nabla \cdot \hat{\mathbf{v}}_{p q}=0 \quad \text { in } \mathbb{R}^{3} \\
\hat{\mathbf{v}}_{p q}(\xi)-\xi_{p} \mathbf{e}_{q}+\delta_{p q} d(\xi) \rightarrow 0 \quad \text { as }|\xi| \rightarrow \infty \\
\hat{p}(\xi) \rightarrow 0 \quad \text { as }|\xi| \rightarrow+\infty .
\end{array}\right.
$$

We define the viscous moment tensor (VMT) $\left(V_{i j}^{p q}\right)_{i, j, p, q=1,2,3}$ by

$$
V_{i j}^{p q}=(\tilde{\mu}-\mu) \int_{B} \nabla \hat{\mathbf{v}}_{p q}:\left(\nabla\left(\xi_{i} \mathbf{e}_{j}\right)+\nabla\left(\xi_{i} \mathbf{e}_{j}\right)^{T}\right) d \xi .
$$

Note that we used the standard notation of the contraction: for matrices $a=\left(a_{i j}\right)$ and $b=\left(b_{i j}\right), a: b=\sum_{i, j} a_{i j} b_{i j}$. It is also worth mentioning that the notion of VMT can be defined in the same manner for two dimensions.

We will realize the notion of VMT as a limit of the corresponding notion for the elasticity, the elastic moment tensor (EMT), from which all the important properties of VMT follow. Before doing that, we rewrite (5.2) and (5.6) using the VMT.

Since $\mathbf{U}_{0}$ is divergence-free, we have

$$
\nabla \mathbf{U}_{0}(z) \xi=\sum_{p, q} \partial_{q} \mathbf{U}_{0}(z)_{p}\left(\xi_{p} \mathbf{e}_{q}-\delta_{p q} d(\xi)\right)
$$

and hence

$$
\hat{\mathbf{v}}_{1}=\sum_{p, q=1}^{3} \partial_{q} \mathbf{U}_{0}(z)_{p} \hat{\mathbf{v}}_{p q}
$$

By (5.2), we have

$$
\mathbf{u}_{0}(x) \approx \mathbf{U}_{0}(z)+\epsilon \sum_{p, q=1}^{3} \partial_{q} \mathbf{U}_{0}(z)_{p} \hat{\mathbf{v}}_{p q}\left(\frac{x-z}{\epsilon}\right) \text { for } x \text { near } z .
$$

By making a further approximation from (5.10), we obtain the following proposition for the inner expansion, the proof of which will be given in the next section.

Proposition 5.1 (Inner expansion). Let $\hat{\mathbf{v}}_{p q}$ be the solution to (5.7). Then, for $x$ away from $\partial \Omega$,

$$
\mathbf{u}_{0}(x)=\mathbf{U}_{0}(x)+\epsilon \hat{\mathbf{v}}_{1}\left(\frac{x-z}{\epsilon}\right)-\nabla \mathbf{U}_{0}(z)(x-z)+O\left(\epsilon^{2}\right) .
$$

We also obtain the following proposition for the outer expansion from (5.6) and the definition (5.8) of the VMT. 
Proposition 5.2 (Outer expansion). Let $\left(V_{i j}^{p q}\right)$ be the VMT defined by (5.8). The following expansion holds uniformly for $x \in \partial \Omega$ :

$$
\begin{aligned}
& \left(\mathbf{u}_{0}-\mathbf{U}_{0}\right)(x) \\
& =\epsilon^{3}\left[\sum_{i, j, p, q, \ell=1}^{3} \mathbf{e}_{\ell} \partial_{j} G_{\ell i}^{\kappa}(x, z) \partial_{q} \mathbf{U}_{0}(z)_{p} V_{i j}^{p q}+|B| \omega^{2} \frac{\rho-\tilde{\rho}}{\mu} \mathbf{G}^{\kappa}(x, z) \mathbf{U}_{0}(z)\right]+O\left(\epsilon^{4}\right),
\end{aligned}
$$

where the differentiation in $\partial_{j} G_{\ell i}^{\kappa}(x, z)$ is with respect to the $z$-variable.

The viscous moment tensor can also be defined using the layer potentials. Let $\left(\hat{\phi}_{p q}, \hat{\psi}_{p q}\right)$ be the unique solution to

$$
\left\{\begin{array}{l}
\mathbf{S}_{B}^{0}\left[\hat{\phi}_{p q}\right]-\mathbf{S}_{B}^{0}\left[\hat{\psi}_{p q}\right]=\xi_{p} \mathbf{e}_{q}-\delta_{p q} d(\xi), \\
\tilde{\mu}\left(-\frac{1}{2}+\left(\mathbf{K}_{B}^{0}\right)^{*}\right)\left[\hat{\phi}_{p q}\right]-\mu\left(\frac{1}{2}+\left(\mathbf{K}_{B}^{0}\right)^{*}\right)\left[\hat{\psi}_{p q}\right]=\mu \frac{\partial}{\partial n}\left(\xi_{p} \mathbf{e}_{q}-\delta_{p q} d(\xi)\right),
\end{array} \quad \text { on } \partial B .\right.
$$

The unique solvability of (5.13) can be proved in exactly the same manner as that of (4.30). Then we have

$$
\hat{\mathbf{v}}_{p q}(\xi)= \begin{cases}\xi_{p} \mathbf{e}_{q}-\delta_{p q} d(\xi)+\mathbf{S}_{B}^{0}\left[\hat{\psi}_{p q}\right](\xi) & \text { in } \mathbb{R}^{3} \backslash \bar{B} \\ \mathbf{S}_{B}^{0}\left[\hat{\phi}_{p q}\right](\xi) & \text { in } B .\end{cases}
$$

Using integration by parts, (5.13), and (5.14), we have

$$
\begin{aligned}
V_{i j}^{p q} & =(\tilde{\mu}-\mu) \int_{B} \nabla \hat{\mathbf{v}}_{p q}:\left(\nabla\left(\xi_{i} \mathbf{e}_{j}\right)+\nabla\left(\xi_{i} \mathbf{e}_{j}\right)^{T}\right) d \xi \\
& =(\tilde{\mu}-\mu) \int_{B} \nabla \hat{\mathbf{v}}_{p q}:\left(\nabla\left(\xi_{i} \mathbf{e}_{j}-\delta_{i j} d(\xi)\right)+\nabla\left(\xi_{i} \mathbf{e}_{j}-\delta_{i j} d(\xi)\right)^{T}\right) d \xi \\
& =(\tilde{\mu}-\mu) \int_{\partial B} \hat{\mathbf{v}}_{p q} \cdot \frac{\partial}{\partial \mathbf{N}}\left(\xi_{i} \mathbf{e}_{j}-\delta_{i j} d(\xi)\right) \\
& =\int_{\partial B}\left(\tilde{\mu} \mathbf{S}_{B}^{0}\left[\hat{\phi}_{p q}\right]-\mu \mathbf{S}_{B}^{0}\left[\hat{\psi}_{p q}\right]-\mu \nabla\left(\xi_{i} \mathbf{e}_{j}-\delta_{i j} d(\xi)\right)\right) \cdot \frac{\partial}{\partial \mathbf{N}}\left(\xi_{i} \mathbf{e}_{j}-\delta_{i j} d(\xi)\right) \\
& =\int_{\partial B}\left(\tilde{\mu}\left(-\frac{1}{2}+\left(\mathbf{K}_{B}^{0}\right)^{*}\right)\left[\hat{\phi}_{p q}\right]-\mu\left(-\frac{1}{2}+\left(\mathbf{K}_{B}^{0}\right)^{*}\right)\left[\hat{\psi}_{p q}\right]-\mu \frac{\partial}{\partial n}\left(\xi_{i} \mathbf{e}_{j}-\delta_{i j} d(\xi)\right)\right) \\
& \cdot\left(\xi_{i} \mathbf{e}_{j}-\delta_{i j} d(\xi)\right) \\
& \mu \int_{\partial B} \hat{\psi}_{p q} \cdot\left(\xi_{i} \mathbf{e}_{j}-\delta_{i j} d(\xi)\right) .
\end{aligned}
$$

In short, we obtained the following lemma.

Lemma 5.3. Let $\left(\hat{\phi}_{p q}, \hat{\psi}_{p q}\right)$ be the unique solution to (5.13). Then

$$
V_{i j}^{p q}=\mu \int_{\partial B} \hat{\psi}_{p q} \cdot\left(\xi_{i} \mathbf{e}_{j}-\delta_{i j} d(\xi)\right), \quad i, j, p, q=1,2,3 .
$$

The notion of the VMT has its counterparts in the conductivity and the elasticity: the electrical polarization tensor (PT) and the elastic moment tensor (EMT). The concept of PT associated with a bounded domain and an isotropic or anisotropic conductivity 
is central in the small volume asymptotic approach. It has been extended to the linear elasticity defining EMT. We refer to [6] for an extensive study of properties and applications of PT and EMT in imaging as well as in the effective medium theory. The viscous moment tensor $V$ is a natural extension of PT and EMT to the Stokes system. As the Stokes system appears as a limiting case of the Lamé system, there is a strong relation between VMT and EMT, which we investigate now.

Let us recall the definition of the elastic moment tensor. Introduce $\mathbf{w}_{p q}$ for $p, q=1,2,3$ as the solution to

$$
\left\{\begin{array}{l}
\mathcal{L}_{\lambda, \mu} \mathbf{w}_{p q}=0 \quad \text { in } \mathbb{R}^{3} \backslash \bar{B}, \\
\mathcal{L}_{\tilde{\lambda}, \tilde{\mu}} \mathbf{w}_{p q}=0 \text { in } B, \\
\left.\mathbf{w}_{p q}\right|_{-}=\left.\mathbf{w}_{p q}\right|_{+} \quad \text { on } \partial B, \\
\left.\frac{\partial \mathbf{w}_{p q}}{\partial \tilde{\nu}}\right|_{-}=\left.\frac{\partial \mathbf{w}_{p q}}{\partial \nu}\right|_{+} \quad \text { on } \partial B, \\
\mathbf{w}_{p q}-\xi_{p} \mathbf{e}_{q} \rightarrow 0 \quad \text { as }|\xi| \rightarrow+\infty .
\end{array}\right.
$$

The elastic moment tensor $M=\left(m_{i j}^{p q}\right)$ associated with the domain $B$ and the pairs of Lamé parameters $(\lambda, \tilde{\lambda} ; \mu, \tilde{\mu})$ is given by

$$
m_{i j}^{p q}=\int_{\partial B}\left[-\frac{\partial\left(\xi_{i} \mathbf{e}_{j}\right)}{\partial \nu}+\frac{\partial\left(\xi_{i} \mathbf{e}_{j}\right)}{\partial \tilde{\nu}}\right] \cdot \mathbf{w}_{p q} d \sigma, \quad i, j, p, q=1,2,3 .
$$

Observe that

$$
-\frac{\partial\left(\xi_{i} \mathbf{e}_{j}\right)}{\partial \nu}+\frac{\partial\left(\xi_{i} \mathbf{e}_{j}\right)}{\partial \tilde{\nu}}=(\tilde{\lambda}-\lambda) \nabla \cdot\left(\xi_{i} \mathbf{e}_{j}\right) \mathbf{N}+(\tilde{\mu}-\mu) \frac{\partial\left(\xi_{i} \mathbf{e}_{j}\right)}{\partial \mathbf{N}} .
$$

In exactly the same manner as in Theorem 3.1, one can prove that

$$
\hat{\mathbf{v}}_{p q}=\lim _{\lambda, \tilde{\lambda} \rightarrow+\infty}\left(\mathbf{w}_{p q}-\frac{\delta_{p q}}{3} \sum_{k} \mathbf{w}_{k k}\right) .
$$

Since

$$
m_{i j}^{p q}-\frac{\delta_{i j}}{3} \sum_{k} m_{k k}^{p q}=(\tilde{\mu}-\mu) \int_{B} \nabla \mathbf{w}_{p q} \cdot\left(\nabla\left(\xi_{i} \mathbf{e}_{j}-\delta_{i j} d(\xi)\right)+\nabla\left(\xi_{j} \mathbf{e}_{i}-\delta_{i j} d(\xi)\right)^{T}\right),
$$

we have the following theorem.

TheOrem 5.4. For $i, j, p, q=1,2,3$,

$$
V_{i j}^{p q}=\lim _{\lambda, \tilde{\lambda} \rightarrow \infty}\left(m_{i j}^{p q}-\frac{\delta_{i j}}{3} \sum_{k=1}^{3} m_{k k}^{p q}-\frac{\delta_{p q}}{3} \sum_{s=1}^{3} m_{i j}^{s s}+\frac{\delta_{i j} \delta_{p q}}{9} \sum_{k, s=1}^{3} m_{k k}^{s s}\right) .
$$

It is known $[7,3]$ that EMT has the symmetry properties: $m_{i j}^{p q}=m_{j i}^{p q}, m_{i j}^{p q}=m_{i j}^{q p}$, and $m_{i j}^{p q}=m_{p q}^{i j}$. Thus as an immediate consequence of (5.17), the following corollary holds.

Corollary 5.5. For $i, j, p, q=1,2,3$,

$$
V_{i j}^{p q}=V_{j i}^{p q}, \quad V_{i j}^{p q}=V_{i j}^{q p}, \quad V_{i j}^{p q}=V_{p q}^{i j}
$$


Moreover, the following holds:

$$
\sum_{p} V_{i j}^{p p}=0 \quad \text { for all } i, j \text { and } \quad \sum_{i} V_{i i}^{p q}=0 \text { for all } p, q .
$$

The relation (5.17) has an interesting interpretation. The VMT $V$ and the EMT $M$ are 4-tensors and can be regarded as linear transformations on the space of symmetric matrices because of their symmetry (5.18). Let $P$ be the orthogonal projection from the space of symmetric matrices onto the space of symmetric matrices of trace zero. Then (5.17) is equivalent to

$$
V=\lim _{\lambda, \tilde{\lambda} \rightarrow \infty} P M P
$$

Including the two-dimensional case into consideration as well, let $\mathbf{I}_{2}$ be the $d \times d$ identity matrix or 2-tensor and $\mathbf{I}_{4}$ the identity 4-tensor. Here $d=2,3$ denotes the space dimension. Then the orthogonal projection $P$ is given by $P=\mathbf{I}_{4}-\frac{1}{d} \mathbf{I}_{2} \otimes \mathbf{I}_{2}$.

The formula (5.20) enables us to compute the VMT from the known formula of the EMT. For example, if $B$ is a disk in two dimensions, then it is known [3] that the EMT is given by

$$
M=|B| \frac{(\lambda+2 \mu)(\tilde{\lambda}+\tilde{\mu}-\lambda-\mu)}{\mu+\tilde{\lambda}+\tilde{\mu}} \mathbf{I}_{2} \otimes \mathbf{I}_{2}+2|B| \frac{\mu(\tilde{\mu}-\mu)\left(\frac{\lambda+3 \mu}{\lambda+\mu}+1\right)}{\mu+\frac{\lambda+3 \mu}{\lambda+\mu} \tilde{\mu}} P,
$$

where $|B|$ is the area of $B$. Thus we have from (5.20) that VMT for a disk $B$ is given by

$$
V=4|B| \mu \frac{(\tilde{\mu}-\mu)}{\tilde{\mu}+\mu} P .
$$

Finally, let us recall the so-called Hashin-Shtrikman bounds for the EMT. Suppose for simplicity that $\tilde{\mu}>\mu$. Set $\tau=\lambda+2 \mu / d, \tilde{\tau}=\tilde{\lambda}+2 \tilde{\mu} / d(\tau$ and $\tilde{\tau}$ are the bulk moduli). From $[28,13]$, we have

$$
\begin{gathered}
\frac{1}{|B|} \operatorname{Tr}(P M P) \leq 2(\tilde{\mu}-\mu)\left[\frac{d^{2}+d-2}{2}-2(\tilde{\mu}-\mu)\left(\frac{d-1}{2 \tilde{\mu}}+\frac{d-1}{d \tilde{\tau}+2(d-1) \tilde{\mu}}\right)\right], \\
|B| \operatorname{Tr}\left(P M^{-1} P\right) \leq \frac{1}{2(\tilde{\mu}-\mu)}\left[\frac{d^{2}+d-2}{2}+2(\tilde{\mu}-\mu)\left(\frac{d-1}{2 \mu}+\frac{d-1}{d \tau+2(d-1) \mu}\right)\right],
\end{gathered}
$$

where $\operatorname{Tr}(C):=\sum_{i, j=1}^{d} C_{i j}^{i j}$ for a 4-tensor $C=\left(C_{i j}^{p q}\right)$. Taking the limits of the HashinShtrikman bounds (5.22) and (5.23) as $\lambda, \tilde{\lambda} \rightarrow+\infty$ shows that $(1 / 2 \mu) V$ satisfies the bounds

$$
\begin{aligned}
\operatorname{Tr}\left(\frac{1}{2 \mu} V\right) & \leq|B|\left(\frac{\tilde{\mu}}{\mu}-1\right)\left((d-1) \frac{\mu}{\tilde{\mu}}+\frac{d(d-1)}{2}\right), \\
\operatorname{Tr}\left(\frac{1}{2 \mu} V\right)^{-1} & \leq \frac{1}{|B|\left(\frac{\tilde{\mu}}{\mu}-1\right)}\left((d-1) \frac{\tilde{\mu}}{\mu}+\frac{d(d-1)}{2}\right) .
\end{aligned}
$$


If we put $k=\tilde{\mu} / \mu$, then in two dimensions $(d=2),(5.24)$ and $(5.25) \mathrm{read}$

$$
\begin{aligned}
\operatorname{Tr}\left(\frac{1}{2 \mu} V\right) & \leq|B|(k-1)\left(\frac{1}{k}+1\right), \\
\operatorname{Tr}\left(\frac{1}{2 \mu} V\right)^{-1} & \leq \frac{k+1}{|B|(k-1)} .
\end{aligned}
$$

It is quite interesting to observe that the quantities on the right-hand sides of $(5.26)$ and (5.27) are exactly the Hashin-Shtrikman bounds for the PT associated with a domain $B$ and conductivity contrast $k$, which were obtained in $[14,28]$. There are several conjectured relations between VMT and PT. We refer the interested reader to [18].

5.3. Justification of the asymptotic expansions. We now prove Propositions 5.1 and 5.2 following the same arguments as those given in [4] for the asymptotic expansion for the Helmholtz equation. It is worth emphasizing that even though only the first-order terms are given in (5.11) and (5.12), our method enables us to derive higher-order terms as well.

Let the function $\mathbf{H}$ be defined by $\mathbf{H}(x)=\mathbf{D}_{\Omega}^{\kappa}[\theta](x)$, where $\theta$ is given by (4.30). Since $D$ is well separated from the boundary $\partial \Omega$, we have, for any $l \in \mathbb{N},\|\mathbf{H}\|_{\mathcal{C}^{l}(\bar{D})} \leq C_{l}$ for some constant $C_{l}$ independent of $\epsilon$. Fix an integer $l$ and define

$$
\mathbf{H}^{l}(x)=\sum_{|\alpha|=0}^{l} \frac{\partial^{\alpha} \mathbf{H}(z)}{\alpha !}(x-z)^{\alpha},
$$

the first $l$ terms of the Taylor expansion of $\mathbf{H}$. Let the pair $\left(\phi^{l}, \psi^{l}\right) \in L^{2}(\partial D)^{3} \times L^{2}(\partial D)^{3}$ (with $\phi^{l}-\psi^{l} \in L_{0}^{2}(\partial D)$ ) be the solution to the following system of integral equations:

$$
\left\{\begin{array}{l}
\mathbf{S}_{D}^{\tilde{\kappa}}\left[\phi^{l}\right]-\mathbf{S}_{D}^{\kappa}\left[\psi^{l}\right]=\mathbf{H}^{l} \quad \text { on } \partial D \\
\tilde{\mu}\left(-\frac{1}{2} I+\left(\mathbf{K}_{D}^{\tilde{\kappa}}\right)^{*}\right)\left[\phi^{l}\right]-\mu\left(\frac{1}{2} I+\left(\mathbf{K}_{D}^{\kappa}\right)^{*}\right)\left[\psi^{l}\right]=\mu \frac{\partial \mathbf{H}^{l}}{\partial n} \quad \text { on } \partial D .
\end{array}\right.
$$

By the linearity of integral equations, one can see that there exists a positive constant $C$ independent of $\epsilon$ such that

$$
\left\|\phi-\phi^{l}\right\|_{L^{2}(\partial D)}+\left\|\psi-\psi^{l}\right\|_{L^{2}(\partial D)} \leq C\left(\epsilon^{-1}|| \mathbf{H}-\mathbf{H}^{l}\left\|_{L^{2}(\partial D)}+\right\| \nabla\left(\mathbf{H}-\mathbf{H}^{l}\right) \|_{L^{2}(\partial D)}\right) .
$$

Since

$$
\begin{aligned}
\left\|\mathbf{H}-\mathbf{H}^{l}\right\|_{L^{2}(\partial D)} & \leq C|\partial D|^{\frac{1}{2}}\left\|\mathbf{H}-\mathbf{H}^{l}\right\|_{L^{\infty}(\partial D)} \\
& \leq C|\partial D|^{\frac{1}{2}} \epsilon^{l+1}\|\mathbf{H}\|_{\mathcal{C}^{l+1}(\bar{D})}
\end{aligned}
$$

and

$$
\left\|\nabla\left(\mathbf{H}-\mathbf{H}^{l}\right)\right\|_{L^{2}(\partial D)} \leq C|\partial D|^{\frac{1}{2}} \epsilon^{l}\|\mathbf{H}\|_{\mathcal{C}^{l+1}(\bar{D})},
$$

we arrive at

$$
\left\|\phi-\phi^{l}\right\|_{L^{2}(\partial D)}+\left\|\psi-\psi^{l}\right\|_{L^{2}(\partial D)} \leq C|\partial D|^{\frac{1}{2}} \epsilon^{l}=C|\partial B|^{\frac{1}{2}} \epsilon^{l+1} .
$$

By Theorem 4.4, we write

$$
\mathbf{u}_{0}(x)=\mathbf{U}_{0}(x)+\mathbf{G}_{D}^{\kappa}\left[\psi^{l}\right](x)+\mathbf{G}_{D}^{\kappa}\left[\psi-\psi^{l}\right](x), \quad x \in \Omega \backslash \bar{D},
$$


and therefore, by estimating for $x$ far away from $z$,

$$
\begin{aligned}
\left|\mathbf{G}_{D}^{\kappa}\left[\psi-\psi^{l}\right](x)\right| & \leq C\left(\int_{\partial D}\left|\mathbf{G}_{D}^{\kappa}(x, y)\right|^{2} d \sigma(y)\right)^{\frac{1}{2}}\left\|\psi-\psi^{l}\right\|_{L^{2}(\partial D)} \\
& \leq C|\partial D|^{\frac{1}{2}} \epsilon^{l+1}=C|\partial B|^{\frac{1}{2}} \epsilon^{l+2}
\end{aligned}
$$

we may conclude that

$$
\mathbf{u}_{0}(x)=\mathbf{U}_{0}(x)+\mathbf{G}_{D}^{\kappa}\left[\psi^{l}\right](x)+O\left(\epsilon^{l+2}\right),
$$

uniformly in $x$ away from $z$.

Recall that $D=\epsilon B+z$. Since $\boldsymbol{\Gamma}^{\epsilon \kappa}(x)=\epsilon \boldsymbol{\Gamma}^{\kappa}(\epsilon x)$, we have, after an obvious scaling,

$$
\mathbf{S}_{D}^{\kappa}[\phi](\epsilon \xi+z)=\epsilon \mathbf{S}_{B}^{\epsilon \kappa}[\tilde{\phi}](\xi), \quad \xi \in \partial B
$$

where $\tilde{\phi}(\xi)=\phi(\epsilon \xi+z)$. Having (5.31) in mind, let the pair $\left(\tilde{\phi}^{j}, \tilde{\psi}^{j}\right) \in L^{2}(\partial B) \times L^{2}(\partial B)$ (with $\tilde{\phi}^{j}-\tilde{\psi}^{j} \in L_{0}^{2}(\partial B)$ ) be the solution to the following system of integral equations:

$$
\left\{\begin{array}{l}
\mathbf{S}_{B}^{\epsilon \tilde{\kappa}}\left[\tilde{\phi}^{j}\right]-\mathbf{S}_{B}^{\epsilon \kappa}\left[\tilde{\psi}^{j}\right]=\sum_{|\alpha|=j} \frac{1}{\alpha !} \partial^{\alpha} \mathbf{H}(z) \xi^{\alpha} \quad \text { on } \partial B \\
\tilde{\mu}\left(-\frac{1}{2} I+\left(\mathbf{K}_{B}^{\epsilon \tilde{\kappa}}\right)^{*}\right)\left[\tilde{\phi}^{j}\right]-\mu\left(\frac{1}{2} I+\left(\mathbf{K}_{B}^{\epsilon \kappa}\right)^{*}\right)\left[\tilde{\psi}^{j}\right]=\mu \frac{\partial}{\partial n} \sum_{|\alpha|=j} \frac{1}{\alpha !} \partial^{\alpha} \mathbf{H}(z) \xi^{\alpha} \quad \text { on } \partial B .
\end{array}\right.
$$

Then, by the change of variables and the linearity of integral equation, we have

$$
\left\{\begin{aligned}
\phi^{l}(z+\epsilon \xi) & =\sum_{j=0}^{l} \epsilon^{j-1} \tilde{\phi}^{j}(\xi) \\
\psi^{l}(z+\epsilon \xi) & =\sum_{j=0}^{l} \epsilon^{j-1} \tilde{\psi}^{j}(\xi)
\end{aligned}\right.
$$

where $\left(\phi^{l}, \psi^{l}\right)$ is the solution of (5.28). Using the Taylor expansion

$$
G^{\kappa}(x, z+\epsilon \xi)=\sum_{|\beta|=0}^{+\infty} \frac{\epsilon^{|\beta|}}{\beta !} \partial_{z}^{\beta} G^{\kappa}(x, z) \xi^{\beta}, \quad \xi \in \partial B
$$

which holds uniformly in $x$ away from $z$, we obtain from (5.30)

$$
\mathbf{u}_{0}(x)=\mathbf{U}_{0}(x)+\sum_{|\beta|=0}^{l} \sum_{j=0}^{l-|\beta|} \frac{\epsilon^{|\beta|+j+1}}{\beta !} \partial_{z}^{\beta} G^{\kappa}(x, z) \int_{\partial B} \xi^{\beta} \tilde{\psi}^{j}(\xi) d \sigma(\xi)+O\left(\epsilon^{l+2}\right),
$$

uniformly in $x$ away from $z$.

Now we calculate the terms in the expansion (5.34) explicitly. First we claim that

$$
\int_{\partial B} \xi^{\beta} \tilde{\psi}^{j}(\xi) d \sigma(\xi)= \begin{cases}-\frac{\tilde{\mu}}{\mu} \epsilon^{2} \tilde{\kappa}^{2}|B| \mathbf{U}_{0}(z)+O\left(\epsilon^{3}\right), & \text { for }(|\beta|, j)=(0,0), \\ O\left(\epsilon^{2}\right), & \text { for }(|\beta|, j)=(0,1),(1,0), \\ \kappa^{2}|B| \mathbf{U}_{0}(z)+O\left(\epsilon^{2}\right), & \text { for }(|\beta|, j)=(0,2) .\end{cases}
$$


To prove (5.35), we first note from (5.32) that

$$
\begin{aligned}
\tilde{\psi}^{j} & =\left(\frac{1}{2} I+\left(\mathbf{K}_{B}^{\epsilon \kappa}\right)^{*}\right)\left[\tilde{\psi}^{j}\right]-\left(-\frac{1}{2} I+\left(\mathbf{K}_{B}^{\epsilon \kappa}\right)^{*}\right)\left[\tilde{\psi}^{j}\right] \\
& =-\frac{\partial}{\partial n} \sum_{|\alpha|=j} \frac{1}{\alpha !} \partial^{\alpha} \mathbf{H}(z) \xi^{\alpha}+\frac{\tilde{\mu}}{\mu}\left(-\frac{1}{2} I+\left(\mathbf{K}_{B}^{\epsilon \tilde{\kappa}}\right)^{*}\right)\left[\tilde{\phi}^{j}\right]-\left(-\frac{1}{2} I+\left(\mathbf{K}_{B}^{\epsilon \kappa}\right)^{*}\right)\left[\tilde{\psi}^{j}\right] .
\end{aligned}
$$

Because of (4.14), it follows from Green's formula (4.11) that

$$
\begin{aligned}
\int_{\partial B} \tilde{\psi}^{j} & =\frac{\tilde{\mu}}{\mu} \int_{B} \Delta \mathbf{S}_{B}^{\epsilon \tilde{\kappa}}\left[\tilde{\phi}^{j}\right]-\int_{B} \Delta \mathbf{S}_{B}^{\epsilon \kappa}\left[\tilde{\psi}^{j}\right]-\sum_{|\alpha|=j} \frac{1}{\alpha !} \partial^{\alpha} \mathbf{H}(z) \int_{\partial B} \frac{\partial \xi^{\alpha}}{\partial n} \\
& =-\frac{\tilde{\mu}}{\mu} \epsilon^{2} \tilde{\kappa}^{2} \int_{B} \mathbf{S}_{B}^{\epsilon \tilde{\kappa}}\left[\tilde{\phi}^{j}\right]+\epsilon^{2} \kappa^{2} \int_{B} \mathbf{S}_{B}^{\epsilon \kappa}\left[\tilde{\psi}^{j}\right]-\sum_{|\alpha|=j} \frac{1}{\alpha !} \partial^{\alpha} \mathbf{H}(z) \int_{\partial B} \frac{\partial \xi^{\alpha}}{\partial n} .
\end{aligned}
$$

According to (4.6) we have

$$
\left(\begin{array}{cc}
\mathbf{S}_{B}^{\epsilon \tilde{\kappa}} & -\mathbf{S}_{B}^{\epsilon \kappa} \\
\tilde{\mu}\left(-\frac{1}{2} I+\left(\mathbf{K}_{B}^{\epsilon \tilde{\kappa}}\right)^{*}\right) & -\mu\left(\frac{1}{2} I+\left(\mathbf{K}_{B}^{\epsilon \kappa}\right)^{*}\right)
\end{array}\right)=A_{0}+\epsilon B+O\left(\epsilon^{2}\right),
$$

where

$$
A_{0}=\left(\begin{array}{cc}
\mathbf{S}_{B}^{0} & -\mathbf{S}_{B}^{0} \\
\tilde{\mu}\left(-\frac{1}{2}+\left(\mathbf{K}_{B}^{0}\right)^{*}\right) & -\mu\left(\frac{1}{2}+\left(\mathbf{K}_{B}^{0}\right)^{*}\right)
\end{array}\right)
$$

and

$$
B=\left(\begin{array}{cc}
-\frac{\tilde{\kappa} \sqrt{-1}}{6 \pi} \int_{\partial B} \cdot d \sigma & -\frac{\kappa \sqrt{-1}}{6 \pi} \int_{\partial B} \cdot d \sigma \\
0 & 0
\end{array}\right) .
$$

From (5.37) we get

$$
\left\|\tilde{\phi}^{j}\right\|_{L^{2}(\partial B)}+\left\|\tilde{\psi}^{j}\right\|_{L^{2}(\partial B)} \leq C
$$

and hence it follows from (5.36) that

$$
\int_{\partial B} \tilde{\psi}^{1}=O\left(\epsilon^{2}\right)
$$

and

$$
\int_{\partial B} \tilde{\psi}^{2}=-\sum_{|\alpha|=2} \frac{1}{\alpha !} \partial^{\alpha} \mathbf{H}(z) \int_{\partial B} \frac{\partial \xi^{\alpha}}{\partial n}+O\left(\epsilon^{2}\right)=\kappa^{2}|B| \mathbf{H}(z)+O\left(\epsilon^{2}\right) .
$$

Equation (5.37) also tells us that

$$
\left(\begin{array}{c}
\tilde{\phi}^{0} \\
\tilde{\psi}^{0}
\end{array}\right)=\left(A_{0}^{-1}-A_{0}^{-1} B A_{0}^{-1}\right)\left(\begin{array}{c}
\mathbf{H}(z) \\
0
\end{array}\right)+O\left(\epsilon^{2}\right)=\left(\begin{array}{c}
\left(\mathbf{S}_{B}^{0}\right)^{-1}[\mathbf{H}(z)] \\
0
\end{array}\right)+O\left(\epsilon^{2}\right) .
$$

It then immediately follows that

$$
\int_{\partial B} \xi_{i} \tilde{\psi}^{0}=O\left(\epsilon^{2}\right)
$$


Using (5.36) we also conclude that

$$
\begin{aligned}
\int_{\partial B} \tilde{\psi}^{0} & =-\frac{\tilde{\mu}}{\mu} \epsilon^{2} \tilde{\kappa}^{2} \int_{B} \mathbf{S}_{B}^{\epsilon \tilde{\kappa}}\left[\tilde{\phi}^{0}\right]+\epsilon^{2} \kappa^{2} \int_{B} \mathbf{S}_{B}^{\epsilon \kappa}\left[\tilde{\psi}^{0}\right] \\
& =-\frac{\tilde{\mu}}{\mu} \epsilon^{2} \tilde{\kappa}^{2}|B| \mathbf{H}(z)+O\left(\epsilon^{3}\right) .
\end{aligned}
$$

By (4.38) we can write

$$
\left(\mathbf{H}-\mathbf{U}_{0}\right)(x)=\mathbf{D}_{\Omega}^{\kappa}\left[\theta-\theta_{0}\right](x), \quad x \in \Omega .
$$

This yields

$$
\left|\nabla\left(\mathbf{H}-\mathbf{U}_{0}\right)(z)\right|+\left|\left(\mathbf{H}-\mathbf{U}_{0}\right)(z)\right| \leq C|| \theta-\theta_{0} \|_{L^{2}(\partial \Omega)},
$$

since $z$ is away from $\partial \Omega$. On the other hand, since $\mathbf{u}_{0}=\mathbf{g}$ on $\partial \Omega$, we have, by (4.30) and (4.38),

$$
\theta-\theta_{0}=\left(\frac{1}{2} I+\mathbf{K}_{\Omega}^{\kappa}\right)^{-1}\left[\left.\mathbf{S}_{D}^{\kappa}[\psi]\right|_{\partial \Omega}\right]
$$

Because

$$
\mathbf{S}_{D}^{\kappa}[\psi]_{i}(x)=\epsilon^{2} \Gamma_{i j}^{\kappa}(x-z) \int_{\partial B} \psi_{j}(z+\epsilon y) d \sigma(y)+O\left(\epsilon^{3}\right), \quad x \in \partial \Omega,
$$

we obtain

$$
\left\|\theta-\theta_{0}\right\|_{L^{2}(\partial \Omega)} \leq C \epsilon^{2}
$$

and hence

$$
\left|\nabla\left(\mathbf{H}-\mathbf{U}_{0}\right)(z)\right|+\left|\left(\mathbf{H}-\mathbf{U}_{0}\right)(z)\right|=O\left(\epsilon^{2}\right) .
$$

By (5.38), (5.39), (5.41), and (5.42), the claim (5.35) follows.

We are now ready to derive the inner and outer expansions. It follows from (5.34) and (5.35) that

$$
\begin{aligned}
& \mathbf{u}_{0}(x)-\mathbf{U}_{0}(x) \\
& =\sum_{k=0}^{2} \epsilon^{k+1} G^{\kappa}(x, z) \int_{\partial B} \tilde{\psi}^{k}(\xi) d \sigma(\xi)+\epsilon^{3} \sum_{i, j, l=1}^{3} \mathbf{e}_{l} \partial_{j} G_{l i}^{\kappa}(x, z) \int_{\partial B} \xi_{j} \mathbf{e}_{i} \cdot \tilde{\psi}^{1}(\xi) d \sigma(\xi) \\
& =\epsilon^{3}\left[\kappa^{2}-\frac{\tilde{\mu} \tilde{\kappa}^{2}}{\mu}\right]|B| G^{\kappa}(x, z) \mathbf{U}_{0}(z)+\epsilon^{3} \sum_{i, j, l=1}^{3} \mathbf{e}_{l} \partial_{j} G_{l i}^{\kappa}(x, z) \int_{\partial B} \xi_{j} \mathbf{e}_{i} \cdot \tilde{\psi}^{1}(\xi) d \sigma(\xi)
\end{aligned}
$$

modulo $O\left(\epsilon^{4}\right)$. To calculate the last term in the above, let $(\hat{\phi}, \hat{\psi})$ be the pair defined in (5.4). Then by the linearity of the solution to the integral equation in (5.4), we have

$$
\hat{\psi}=\sum_{p, q=1}^{3} \partial_{q} \mathbf{U}_{0}(z)_{q} \hat{\psi}_{p q} .
$$


Moreover, we have from (5.37)

$$
\begin{aligned}
\left(\begin{array}{c}
\tilde{\phi}^{1} \\
\tilde{\psi}^{1}
\end{array}\right) & =A_{0}^{-1}\left(\begin{array}{c}
\nabla \mathbf{H}(z) \xi \\
\mu \frac{\partial}{\partial n}(\nabla \mathbf{H}(z) \xi)
\end{array}\right)+O(\epsilon) \\
& =A_{0}^{-1}\left(\begin{array}{c}
\nabla \mathbf{U}_{0}(z) \xi \\
\mu \frac{\partial}{\partial n}\left(\nabla \mathbf{U}_{0}(z) \xi\right)
\end{array}\right)+O(\epsilon)=\left(\begin{array}{c}
\hat{\phi} \\
\hat{\psi}
\end{array}\right)+O(\epsilon) .
\end{aligned}
$$

It then follows from $(5.46),(5.47)$, and (5.15) that

$$
\begin{aligned}
& \int_{\partial B} \xi_{j} \mathbf{e}_{i} \cdot \tilde{\psi}^{1}(\xi) d \sigma(\xi) \\
& =\int_{\partial B} \xi_{j} \mathbf{e}_{i} \cdot \hat{\psi}(\xi) d \sigma(\xi)+O(\epsilon) \\
& =\sum_{p, q=1}^{3} \partial_{q} \mathbf{U}_{0}(z)_{p} \int_{\partial B} \xi_{j} \mathbf{e}_{i} \cdot \hat{\psi}_{p q}(\xi) d \sigma(\xi)+O(\epsilon) \\
& =\sum_{p, q=1}^{3} \partial_{q} \mathbf{U}_{0}(z)_{p} V_{i j}^{p q}+\sum_{p, q=1}^{3} \delta_{i j} \partial_{q} \mathbf{U}_{0}(z)_{p} \int_{\partial B} d(\xi) \cdot \hat{\psi}_{p q}(\xi) d \sigma(\xi)+O(\epsilon) .
\end{aligned}
$$

Note that

$$
\sum_{i, j, l=1}^{3} \mathbf{e}_{l} \partial_{j} G_{l i}^{\kappa}(x, z) \sum_{p, q=1}^{3} \delta_{i j} \partial_{q} \mathbf{U}_{0}(z)_{p} \int_{\partial B} d(\xi) \cdot \hat{\psi}_{p q}(\xi) d \sigma(\xi)=0
$$

since $\mathbf{G}^{\kappa}$ is divergence-free. Substituting (5.49) into (5.45) yields (5.12), and Proposition 5.2 is then proved.

We now drive the inner expansion (5.11). Having the representation (4.29) of $\mathbf{u}_{0}$ in mind, we first observe that

$$
\mathbf{S}_{D}^{\tilde{\kappa}}[\phi]=\mathbf{S}_{D}^{\tilde{\kappa}}\left[\phi^{1}\right]+O\left(\epsilon^{2}\right),
$$

which follows from (5.29) with $l=1$. Further, by (4.6) and (5.33), we have, after obvious scaling,

$$
\mathbf{S}_{D}^{\tilde{\kappa}}\left[\phi^{1}\right](z+\epsilon \xi)=\epsilon \mathbf{S}_{B}^{\epsilon \tilde{\kappa}}\left[\frac{1}{\epsilon} \tilde{\phi}^{0}+\tilde{\phi}^{1}\right](\xi)=\epsilon \mathbf{S}_{B}^{0}\left[\frac{1}{\epsilon} \tilde{\phi}^{0}+\tilde{\phi}^{1}\right](\xi)+O\left(\epsilon^{2}\right)
$$

By (5.40) and (5.47), we obtain

$$
\mathbf{S}_{B}^{0}\left[\tilde{\phi}^{0}\right](\xi)=\mathbf{H}(z), \quad \mathbf{S}_{B}^{0}\left[\tilde{\phi}^{1}\right](\xi)=\mathbf{S}_{B}^{0}[\hat{\phi}](\xi)+O(\epsilon) .
$$

It then follows from (5.3) and (5.44) that for $x \in D$,

$$
\mathbf{u}_{0}(x)=\mathbf{S}_{D}^{\tilde{\kappa}}[\phi](x)=\mathbf{U}_{0}(z)+\epsilon \mathbf{S}_{B}^{0}[\hat{\phi}]\left(\frac{x-z}{\epsilon}\right)+O\left(\epsilon^{2}\right) .
$$

Similarly, we have

$$
\mathbf{S}_{D}^{\kappa}\left[\psi^{1}\right](z+\epsilon \xi)=\epsilon \mathbf{S}_{B}^{0}[\hat{\psi}](\xi)+O\left(\epsilon^{2}\right),
$$


and hence, for $x=z+\epsilon \xi \notin D$,

$$
\begin{aligned}
\mathbf{u}_{0}(x) & =\mathbf{H}(x)+\mathbf{S}_{D}^{\kappa}\left[\psi^{1}\right](z+\epsilon \xi)+O\left(\epsilon^{2}\right) \\
& =\mathbf{U}_{0}(x)+\epsilon \hat{\mathbf{v}}_{1}\left(\frac{x-z}{\epsilon}\right)-\nabla \mathbf{U}_{0}(z)(x-z)+O\left(\epsilon^{2}\right),
\end{aligned}
$$

where the last equality holds because of (5.3). Combining (5.51) and (5.52) yields (5.11) and Proposition 5.1 is then proved.

6. Reconstruction method. Based on the inner asymptotic expansion (5.11) of $\delta \mathbf{u}(:=\mathbf{u}-\mathbf{U})$ of the perturbations in the displacement field that are due to the anomaly, we propose a new reconstruction method of binary level set type.

The original level set method was proposed by Osher and Sethian [33] for tracing interfaces between different phases of fluid flow. In [16], Chan and Tai have performed a study on the conductivity inverse problem using continuous level set functions (distance functions) in a standard level set formulation. The use of binary represented level set functions for solving that problem has been proposed in [32].

The first step for our reconstruction procedure is to locate the anomaly. This can be done using the measurements $\delta \mathbf{u}(:=\mathbf{u}-\mathbf{U})$ of the perturbations in the displacement field far away from the anomaly in much the same spirit as the works in $[7,25]$ and recent text [3].

Suppose that $z$ is reconstructed. Since the representation $D=z+\epsilon B$ is not unique, we can fix $\epsilon$. Following [32], we use a binary level set representation $f$ of the scaled domain $B$ :

$$
f(x)= \begin{cases}1, & x \in B \\ -1, & x \in \mathbb{R}^{3} \backslash \bar{B}\end{cases}
$$

Let

$$
2 h(x)=\tilde{\mu}\left(f\left(\frac{x-z}{\epsilon}\right)+1\right)-\mu\left(f\left(\frac{x-z}{\epsilon}\right)-1\right),
$$

and let $\beta$ be a regularization parameter. Then the second step is to fix a window $W$ (a sphere containing $z$ ) and solve the following constrained minimization problem:

$$
\min _{\tilde{\mu}, f} L(f, \tilde{\mu})=\frac{1}{2}\left\|\delta \mathbf{u}(x)-\epsilon \hat{\mathbf{v}}_{1}\left(\frac{x-z}{\epsilon}\right)+\nabla \mathbf{U}_{0}(z)(x-z)\right\|_{L^{2}(W)}^{2}+\beta \int_{W}|\nabla h(x)| d x
$$

subject to (5.1). Here, $\int_{W}|\nabla h| d x$ is the total variation of the shear modulus, and $|\nabla h|$ is understood as a measure:

$$
\int_{W}|\nabla h|=\sup \left\{\int_{W} h \nabla \cdot \mathbf{v} d x, \mathbf{v} \in \mathcal{C}_{0}^{1}(W) \text { and }|\mathbf{v}| \leq 1 \text { in } W\right\} .
$$

This regularization indirectly controls both the length of the level set curves and the jumps in the coefficients.

The local character of the method is due to the decay of $\hat{\mathbf{v}}_{1}((\cdot-z) / \epsilon)-\nabla \mathbf{U}(z)(\cdot-z) / \epsilon$ away from $z$. Replacing $W$ by $\Omega$ in the above formulation does not lead to a better reconstruction of the shape and the shear modulus of the anomaly. This is one of the main features of our method.

The minimization problem (6.3) corresponds to a minimization with respect to $\tilde{\mu}$ followed by a step of minimization with respect to $f$. The minimization steps are over 
the set of $\tilde{\mu}$ and $f$ and can be performed using a gradient-based method with a line search. Of importance to us are the optimal bounds satisfied by the viscous moment tensor $V$. We should check for each step whether the bounds (5.24) and (5.25) on $V$ are satisfied. In the case when they are not, we have to restate the value of $\tilde{\mu}$. Another way to deal with (5.24) and (5.25) is to introduce them into the minimization problem (6.3) as a constraint. Set $A=\operatorname{Tr}(V)$ and $B=\operatorname{Tr}\left(V^{-1}\right)$ and suppose for simplicity that $\tilde{\mu}>\mu$. Then (5.24) and (5.25) can be rewritten (when $d=3$ ) as follows:

$$
\left\{\begin{array}{l}
A \leq 2(\tilde{\mu}-\mu)\left(3+\frac{2 \mu}{\tilde{\mu}}\right)|D|, \\
\frac{2 \mu(\tilde{\mu}-\mu)}{3 \mu+2 \tilde{\mu}}|D| \leq B^{-1} .
\end{array}\right.
$$

In what follows we extend the definition of the Lagrangian $L(f, \tilde{\mu})$ to include not only binary functions but also functions of bounded variation and then define an augmented Lagrangian functional which incorporates the fact that the solution we seek is a binary function. To find a discrete saddle point for this augmented Lagrangian functional, the Uzawa algorithm for variational binary level set methods can be used. The convergence analysis follows [17].

6.1. Variational formulation of (5.1). Here we derive a variational formulation of (5.1) for the purpose of extending the definition of the Lagrangian $L(f, \tilde{\mu})$ to include functions of bounded variation.

Recall that the solution $\hat{\mathbf{v}}_{1}$ of equation (5.1) defines the first-order correction in the inner expansion of the displacement field. Set for the sake of simplicity $z$ at the origin. Let $\mathcal{O}$ be a sphere of radius $R$ such that $B \subset \subset \mathcal{O}$. Define $H_{\text {div }}(\mathcal{O})=\left\{\mathbf{w} \in H^{1}(\mathcal{O})\right.$ : $\nabla \cdot \mathbf{w}=0\}$. Following [21, 22], introduce the transparent operator $T$ on $H^{1 / 2}(\partial \mathcal{O})$ defined by

$$
T(\mathbf{g})=-\left(p \mathbf{N}+\mu \frac{\partial \mathbf{w}}{\partial \mathbf{N}}\right) \quad \text { on } \partial \mathcal{O},
$$

where $\mathbf{w}$ is the solution to

$$
\left\{\begin{array}{l}
\mu \Delta \mathbf{w}+\nabla p=0 \quad \text { in } \mathbb{R}^{3} \backslash \overline{\mathcal{O}} \\
\mathbf{w}=\mathbf{g} \quad \text { on } \partial \mathcal{O} \\
\nabla \cdot \mathbf{w}=0 \quad \text { in } \mathbb{R}^{3} \backslash \overline{\mathcal{O}} \\
\mathbf{w}(\xi) \rightarrow 0 \quad|\xi| \rightarrow+\infty \\
p(\xi) \rightarrow 0 \quad|\xi| \rightarrow+\infty
\end{array}\right.
$$

According to [22], the operator $T$ is continuous from $H^{1 / 2}(\partial \mathcal{O})$ to $H^{-1 / 2}(\partial \mathcal{O})$. It is self-adjoint, and

$$
\langle T \mathbf{g}, \mathbf{g}\rangle_{\frac{1}{2},-\frac{1}{2}}=\|\mathbf{g}\|_{H^{1 / 2}(\partial \mathcal{O})}^{2},
$$

where $\langle\cdot, \cdot\rangle_{\frac{1}{2},-\frac{1}{2}}$ denotes the duality pair between $H^{1 / 2}(\partial \mathcal{O})$ and $H^{-1 / 2}(\partial \mathcal{O})$.

Writing

$$
(\tilde{\mu}-\mu) \int_{\partial B}\left(\nabla \mathbf{U}(0)+\nabla \mathbf{U}(0)^{T}\right) \mathbf{N}(\xi) \cdot \mathbf{w}(\xi) d \sigma(\xi)=-\int_{\mathcal{O}} \nabla \hat{h}(\xi) \cdot\left(\nabla \mathbf{U}(0)+\nabla \mathbf{U}(0)^{T}\right) \mathbf{w}(\xi) d \xi,
$$


it is easy to see that $\hat{\mathbf{v}}_{1}(\xi)-\nabla \mathbf{U}(0) \xi$ is the solution to the variational formulation: find $\mathbf{v} \in H_{\text {div }}(\mathcal{O})$ such that

$$
\begin{aligned}
\int_{\mathcal{O}} \hat{h}(\xi)\left(\nabla \mathbf{v}(\xi)+\nabla \mathbf{v}(\xi)^{T}\right): \nabla \mathbf{w}(\xi) d \xi & +\langle T \mathbf{v}, \mathbf{w}\rangle_{\frac{1}{2},-\frac{1}{2}} \\
& =\int_{\mathcal{O}} \nabla \hat{h}(\xi) \cdot\left(\nabla \mathbf{U}(0)+\nabla \mathbf{U}(0)^{T}\right) \mathbf{w}(\xi) d \xi
\end{aligned}
$$

for all $\mathbf{w} \in H_{\operatorname{div}}(\mathcal{O})$, where $\hat{h}$ is given by

$$
\hat{h}(\xi)= \begin{cases}\tilde{\mu}, & \xi \in B \\ \mu, & \xi \in \mathcal{O} \backslash \bar{B} .\end{cases}
$$

6.2. Lagrangian formulation of the problem. We now derive an augmented Lagrangian formulation of the problem. We first simplify (6.3). Notice that $L$ actually depends on $h$, and according to the chain rule we have

$$
\frac{\partial L}{\partial f}=\frac{\partial L}{\partial h} \frac{\partial h}{\partial f}, \quad \frac{\partial L}{\partial \tilde{\mu}}=\frac{\partial L}{\partial h} \frac{\partial h}{\partial \tilde{\mu}} .
$$

Thus we may consider $L$ as a function of $h$ only. Set $\mathcal{O}=\frac{1}{\epsilon} W$ and $\widetilde{\mathbf{v}}(\xi)=\hat{\mathbf{v}}_{1}(\xi)-\nabla \mathbf{U}(0) \xi$. By a change of variables, (6.3) becomes:

$$
\min _{\hat{h}} L^{0}(\hat{h})=\frac{\epsilon}{2}\|\delta \mathbf{u}(\epsilon \xi)-\epsilon \widetilde{\mathbf{v}}(\xi)\|_{L^{2}(\mathcal{O})}^{2}+\beta \int_{\mathcal{O}}|\nabla \hat{h}(\xi)| d \xi
$$

subject to (6.5). We should emphasize that this functional depends on $\hat{h}$, both through the regularization term and through the solution $\widetilde{\mathbf{v}}$ of (6.5) itself.

Next, we relax the definition of the anomaly by reformulating the definition of $f$. Notice that (6.5) can be extended to any $\hat{h}$ of bounded variation, which is exactly the idea behind a Lagrangian formulation. So we define the anomaly by $f \in B V(\mathcal{O})$, the space of functions of bounded variation on $\mathcal{O}$, and let $\hat{h}$ be defined through (6.2), namely

$$
2 \hat{h}(\xi)=\tilde{\mu}(f(\xi)+1)-\mu(f(\xi)-1) .
$$

Set $K(f)=f^{2}-1$. Then $f$, being a binary function, is equivalent to $K(f)=0$. We shall thus replace (6.3) by the relaxed formulation, referred to as the augmented Lagrangian formulation:

$$
\min _{\hat{h}, \lambda} L(\hat{h}, \lambda)=\frac{\epsilon}{2}\|\delta \mathbf{u}(\epsilon \xi)-\epsilon \widetilde{\mathbf{v}}(\xi)\|_{L^{2}(\mathcal{O})}^{2}+\beta \int_{\mathcal{O}}|\nabla \hat{h}(\xi)| d \xi+\lambda \int_{\mathcal{O}} K(f)^{2}(\xi) d \xi
$$

subject to $(6.5)$, where $f=(2 \hat{h}-\tilde{\mu}-\mu) /(\tilde{\mu}-\mu)$, the minimization is over $\hat{h} \in B V(\mathcal{O})$, and $\lambda \in \mathbb{R}^{+}$is the Lagrangian multiplier.

In order to investigate the saddle points of this functional, we shall compute its derivative with respect to the variable $\hat{h}$. For calculating the gradient of this functional, we use the adjoint state method, which requires us to use the variational form (6.5). 
6.3. Adjoint state method. The functional $L^{0}$ in (6.6) depends on $\widetilde{\mathbf{v}}$ which itself depends on $\hat{h}$. Let us write $\widetilde{\mathbf{v}}=\widetilde{\mathbf{v}}(\hat{h})$. We define for $\hat{h} \in B V(\mathcal{O}), \mathbf{v}, \mathbf{w} \in H_{\text {div }}(\mathcal{O})$ :

$$
\begin{aligned}
L^{1}(\hat{h}, \mathbf{v}, \mathbf{w})= & \frac{\epsilon}{2}\|\delta \mathbf{u}(\epsilon \xi)-\epsilon \mathbf{v}(\xi)\|_{L^{2}(\mathcal{O})}^{2}+\beta \int_{\mathcal{O}}|\nabla \hat{h}(\xi)| d \xi \\
& +\int_{\mathcal{O}} \hat{h}(\xi)\left(\nabla \mathbf{v}(\xi)+\nabla \mathbf{v}(\xi)^{T}\right): \nabla \mathbf{w}(\xi) d \xi+\langle T \mathbf{v}, \mathbf{w}\rangle_{\frac{1}{2},-\frac{1}{2}} \\
& -\int_{\mathcal{O}} \nabla \hat{h}(\xi) \cdot\left(\nabla \mathbf{U}(0)+\nabla \mathbf{U}(0)^{T}\right) \mathbf{w}(\xi) d \xi .
\end{aligned}
$$

Since $\widetilde{\mathbf{v}}$ satisfies $(6.5)$, it follows that for all $\mathbf{w} \in H_{\operatorname{div}}(\mathcal{O})$,

$$
L^{0}(\hat{h})=L^{1}(\hat{h}, \widetilde{\mathbf{v}}, \mathbf{w})
$$

Thus for any $\eta \in B V(\mathcal{O})$, the derivative $\frac{\partial L^{0}}{\partial \hat{h}}$ in the direction $\eta$ is given by

$$
\left\langle\frac{\partial L^{0}}{\partial \hat{h}}, \eta\right\rangle=\left\langle\frac{\partial L^{1}}{\partial \hat{h}}(\hat{h}, \widetilde{\mathbf{v}}, \mathbf{w}), \eta\right\rangle+\left\langle\frac{\partial L^{1}}{\partial \mathbf{v}}(\hat{h}, \widetilde{\mathbf{v}}, \mathbf{w}), \frac{\partial \widetilde{\mathbf{v}}}{\partial \hat{h}}[\eta]\right\rangle,
$$

where

$$
\frac{\partial \widetilde{\mathbf{v}}}{\partial \hat{h}}[\eta]=\lim _{\epsilon \rightarrow 0} \frac{\widetilde{\mathbf{v}}(\hat{h}+\epsilon \eta)-\widetilde{\mathbf{v}}(\hat{h})}{\epsilon}
$$

which exists in $H_{\text {div }}(\mathcal{O})$. This formula gives access to the derivative $\frac{\partial L^{0}}{\partial \hat{h}}$, as soon as $\mathbf{w}=\widetilde{\mathbf{w}}$, given by

$$
\frac{\partial L^{1}}{\partial \mathbf{v}}(\hat{h}, \widetilde{\mathbf{v}}, \widetilde{\mathbf{w}})=0
$$

Suppose (6.10) is fulfilled. Differentiating with respect to $\hat{h}$, we get

$$
\begin{aligned}
\left\langle\frac{\partial L^{0}}{\partial \hat{h}}, \eta\right\rangle & =-\beta \int_{\mathcal{O}} \nabla \cdot\left(\frac{\nabla \hat{h}}{|\nabla \hat{h}|}\right)(\xi) \eta(\xi) d \xi+\int_{\mathcal{O}} \eta(\xi)\left(\nabla \widetilde{\mathbf{v}}(\xi)+\nabla \widetilde{\mathbf{v}}(\xi)^{T}\right): \nabla \widetilde{\mathbf{w}}(\xi) d \xi \\
& +\int_{\mathcal{O}} \eta(\xi) \nabla \cdot\left(\nabla \mathbf{U}(0)+\nabla \mathbf{U}(0)^{T}\right) \widetilde{\mathbf{w}}(\xi) d \xi
\end{aligned}
$$

or equivalently,

$$
\frac{\partial L^{0}}{\partial \hat{h}}=-\beta \nabla \cdot\left(\frac{\nabla \hat{h}}{|\nabla \hat{h}|}\right)+\left(\nabla \widetilde{\mathbf{v}}(\xi)+\nabla \widetilde{\mathbf{v}}(\xi)^{T}\right): \nabla \widetilde{\mathbf{w}}+\nabla \cdot\left(\nabla \mathbf{U}(0)+\nabla \mathbf{U}(0)^{T}\right) \widetilde{\mathbf{w}},
$$

$\widetilde{\mathbf{v}}$ being defined by the variational formulation (6.5) and $\widetilde{\mathbf{w}}$ being determined as the adjoint state, by $\frac{\partial L^{1}}{\partial \mathbf{v}}(\hat{h}, \widetilde{\mathbf{v}}, \widetilde{\mathbf{w}})=0$. This is equivalent to the following variational formulation: find $\widetilde{\mathbf{w}} \in H_{\operatorname{div}}(\mathcal{O})$ such that for all $\mathbf{z} \in H_{\operatorname{div}}(\mathcal{O})$,

$$
\int_{\mathcal{O}} \hat{h}(x) \nabla \widetilde{\mathbf{w}}(\xi):\left(\nabla \mathbf{z}(\xi)+\nabla \mathbf{z}(\xi)^{T}\right) d \xi+\langle T \widetilde{\mathbf{w}}, \mathbf{z}\rangle_{\frac{1}{2},-\frac{1}{2}}=\epsilon^{2} \int_{\mathcal{O}} \mathbf{z}(\xi) \cdot(\delta \mathbf{u}(\epsilon \xi)-\epsilon \widetilde{\mathbf{v}}(\xi)) d \xi .
$$


6.4. Lagrangian saddle points-algorithm. We investigate the saddle points of the Lagrangian functional $L^{0}$ and evolve the different parameters from an initial guess $f^{0}, \tilde{\mu}^{0}, \lambda^{0}$ toward those saddle points. We use a gradient type method, and thus we summarize the derivatives of the Lagrangian functional $L^{0}: f$ and $\tilde{\mu}$ being fixed, $\hat{h}$ is fixed and we solve (6.5) and (6.12) to compute $\widetilde{\mathbf{v}}$ and $\widetilde{\mathbf{w}}$.

According to the chain rule, we obtain that

$$
\left\{\begin{array}{l}
\frac{\partial L^{0}}{\partial f}(f, \tilde{\mu})=\frac{\partial L^{0}}{\partial \hat{h}}\left[\frac{\partial \hat{h}}{\partial f}\right] \\
=\frac{(\tilde{\mu}-\mu)}{2}\left(-\beta \nabla \cdot\left(\frac{\nabla \hat{h}}{|\nabla \hat{h}|}\right)+\left(\nabla \widetilde{\mathbf{v}}(\xi)+\nabla \widetilde{\mathbf{v}}(\xi)^{T}\right): \nabla \widetilde{\mathbf{w}}+\nabla \cdot\left(\nabla \mathbf{U}(0)+\nabla \mathbf{U}(0)^{T}\right) \widetilde{\mathbf{w}}\right) \\
\frac{\partial L^{0}}{\partial \tilde{\mu}}(f, \tilde{\mu})=\frac{\partial L^{0}}{\partial \hat{h}}\left[\frac{\partial \hat{h}}{\partial \tilde{\mu}}\right] \\
=\left\langle-\beta \nabla \cdot\left(\frac{\nabla \hat{h}}{|\nabla \hat{h}|}\right)+\left(\nabla \widetilde{\mathbf{v}}(\xi)+\nabla \widetilde{\mathbf{v}}(\xi)^{T}\right): \nabla \widetilde{\mathbf{w}}+\nabla \cdot\left(\nabla \mathbf{U}(0)+\nabla \mathbf{U}(0)^{T}\right) \widetilde{\mathbf{w}}, \frac{f+1}{2}\right\rangle \\
\frac{\partial L}{\partial f}=\frac{\partial L^{0}}{\partial f}+4 \lambda f K(f) \\
\frac{\partial L}{\partial \tilde{\mu}}=\frac{\partial L^{0}}{\partial \tilde{\mu}} .
\end{array}\right.
$$

The parameters evolve following the gradient direction $\nabla L$, in order to minimize the Lagrangian. One introduces an artificial time variable and states the following timeevolutive PDE:

$$
\frac{\partial f}{\partial t}=-\frac{\partial L}{\partial f}, \quad \frac{\partial \tilde{\mu}}{\partial t}=-\frac{\partial L}{\partial \tilde{\mu}},
$$

which leads to the following discrete update scheme:

$$
\begin{aligned}
& f^{k+1}=f^{k}-\Delta t_{f} \cdot \frac{\partial L}{\partial f}\left(f^{k}, \tilde{\mu}^{k}, \lambda^{k}\right), \\
& \tilde{\mu}^{k+1}=\tilde{\mu}^{k}-\Delta t_{\tilde{\mu}} \cdot \frac{\partial L}{\partial \tilde{\mu}}\left(f^{k+1}, \tilde{\mu}^{k}, \lambda^{k}\right),
\end{aligned}
$$

where $\Delta t_{f}$ and $\Delta t_{\tilde{\mu}}$ are well-chosen time steps. To update $\lambda$ we perform as suggested in $[32]$ :

$$
\lambda^{k+1}=\lambda^{k}+\Delta_{\lambda} K\left(f^{k+1}\right),
$$

where $\Delta_{\lambda}$ is a fixed parameter. We perform this update $k \rightarrow k+1$ until the change in these variables, or the differentials of the Lagrangian, is small enough. To conclude, we draw the main features of a classical gradient descent algorithm:

- Initialization: define $f^{0}, \tilde{\mu}^{0}, \lambda^{0}$ and set $k=0$;

- update $f$ :

compute $\widetilde{\mathbf{v}}, \widetilde{\mathbf{w}}$ using the variational formulations (6.5) and (6.12);

update $f^{k} \rightarrow f^{k+1}$ using (6.14);

- update $\tilde{\mu}$ : 
compute $\widetilde{\mathbf{v}}, \widetilde{\mathbf{w}}$ using the variational formulation (6.5) and (6.12) (with the updated value of $\left.f^{k+1}\right)$;

update $\tilde{\mu}^{k} \rightarrow \tilde{\mu}^{k+1}$ using (6.14);

- update $\lambda$ using (6.15);

- estimate $\|\nabla L\|$ and do $k \rightarrow k+1$ until $\|\nabla L\|$ is small enough.

7. Some extensions. In this section we first briefly discuss how to extend our approach to detect the shape and the anisotropic shear modulus of an anisotropic anomaly. In general, the viscoelastic parameters of biological tissue show anisotropic properties, i.e., the local value of elasticity is different in the different spatial directions [36].

7.1. Anisotropic anomaly. Let $A$ and $\tilde{A}$ be two positive-definite symmetric matrices with $A \neq \tilde{A}$. Suppose that $\tilde{A}-A$ is either positive-definite or negative-definite. Introduce

$$
\frac{\partial}{\partial \mathbf{N}_{A}}=\frac{1}{2} A\left(\nabla+\nabla^{T}\right) \cdot \mathbf{N}, \quad \frac{\partial}{\partial \mathbf{N}_{\tilde{A}}}=\frac{1}{2} \tilde{A}\left(\nabla+\nabla^{T}\right) \cdot \mathbf{N} .
$$

Suppose that the shear modulus takes the anisotropic form $A$ and $\tilde{A}$ in $\Omega \backslash \bar{D}$ and $D$, respectively.

Following the same lines as in the derivation of the modified Stokes system in Section 3 , we can reduce the anisotropic elasticity system to an anisotropic Stokes system. Define $\hat{\mathbf{w}}$ as the solution to

$$
\left\{\begin{array}{l}
A \Delta \hat{\mathbf{w}}+\nabla \hat{p}=0 \quad \text { in } \mathbb{R}^{3} \backslash \bar{B}, \\
\tilde{A} \Delta \hat{\mathbf{w}}+\nabla \hat{p}=0 \quad \text { in } B, \\
\left.\hat{\mathbf{w}}\right|_{-}-\left.\hat{\mathbf{w}}\right|_{+}=0 \quad \text { on } \partial B, \\
\left.\left(\hat{p} \mathbf{N}+\frac{\partial \hat{\mathbf{w}}}{\partial \mathbf{N}_{\tilde{A}}}\right)\right|_{-}-\left.\left(\hat{p} \mathbf{N}+\frac{\partial \hat{\mathbf{w}}}{\partial \mathbf{N}_{A}}\right)\right|_{+}=0 \quad \text { on } \partial B, \\
\nabla \cdot \hat{\mathbf{w}}=0 \quad \text { in } \mathbb{R}^{3}, \\
\hat{\mathbf{w}}(\xi)-\nabla \mathbf{U}_{0}(z) \xi \rightarrow 0 \quad|\xi| \rightarrow+\infty, \\
\hat{p}(\xi) \rightarrow 0 \quad|\xi| \rightarrow+\infty .
\end{array}\right.
$$

To reconstruct the anisotropic shear modulus $\tilde{A}$ and the shape of the elastic anomaly $D$, we can prove by exactly the same arguments as those given for the isotropic case that it suffices to replace the minimization problem (6.3) with

$$
\min _{\tilde{A}, f} \frac{1}{2}\left\|\delta \mathbf{u}(x)-\epsilon \hat{\mathbf{w}}\left(\frac{x-z}{\epsilon}\right)+\nabla \mathbf{U}_{0}(z)(x-z)\right\|_{L^{2}(W)}^{2}+\beta \int_{W}|\nabla h(x)| d x,
$$

subject to $(7.1)$.

7.2. Transient elastography. The results and techniques of this paper work for transient elastography. The time-dependent elasticity system in the presence of the anomaly 
$D$ is given by

$$
\left\{\begin{array}{l}
\mathcal{L}_{\lambda, \mu} \mathbf{u}-\rho \partial_{t}^{2} \mathbf{u}=0 \quad \text { in } \Omega \backslash \bar{D} \times[0, T] \\
\mathcal{L}_{\tilde{\lambda}, \tilde{\mu}} \mathbf{u}-\tilde{\rho} \partial_{t}^{2} \mathbf{u}=0 \quad \text { in } D \times[0, T] \\
\left.\mathbf{u}\right|_{-}=\left.\mathbf{u}\right|_{+} \text {on } \partial D \times[0, T] \\
\left.\frac{\partial \mathbf{u}}{\partial \tilde{\nu}}\right|_{-}=\left.\frac{\partial \mathbf{u}}{\partial \nu}\right|_{+} \quad \text { on } \partial D \times[0, T] \\
\left.\mathbf{u}\right|_{\partial \Omega \times[0, T]}=\mathbf{g}, \\
\mathbf{u}(x, 0)=\mathbf{u}_{0}(x), \partial_{t} \mathbf{u}(x, 0)=\mathbf{u}_{1}(x) \quad \text { in } \Omega
\end{array}\right.
$$

By combining the arguments of this paper with those in [5], we can prove without any new difficulty that the following approximation holds:

$$
\delta \mathbf{u}(x, t) \approx \epsilon \hat{\mathbf{v}}\left(\frac{x-z}{\epsilon}, t\right)-\nabla \mathbf{U}_{0}(z, t)(x-z) \quad \text { for } x \text { near } z, \quad \text { and } t \in[0, T],
$$

where $\hat{\mathbf{v}}$ is the solution to $(5.1)$ with $\nabla \mathbf{U}_{0}(z)$ replaced with $\nabla \mathbf{U}_{0}(z, t)$, i.e.,

$$
\left\{\begin{array}{l}
\mu \Delta \hat{\mathbf{v}}+\nabla \hat{p}=0 \quad \text { in } \mathbb{R}^{3} \backslash \bar{B} \\
\tilde{\mu} \Delta \hat{\mathbf{v}}+\nabla \hat{p}=0 \quad \text { in } B \\
\left.\hat{\mathbf{v}}\right|_{-}-\left.\hat{\mathbf{v}}\right|_{+}=0 \quad \text { on } \partial B \\
\left.\left(\hat{p} \mathbf{N}+\tilde{\mu} \frac{\partial \hat{\mathbf{v}}}{\partial \mathbf{N}}\right)\right|_{-}-\left.\left(\hat{p} \mathbf{N}+\mu \frac{\partial \hat{\mathbf{v}}}{\partial \mathbf{N}}\right)\right|_{+}=0 \quad \text { on } \partial B \\
\nabla \cdot \hat{\mathbf{v}}=0 \quad \text { in } \mathbb{R}^{3} \\
\hat{\mathbf{v}}(\xi)-\nabla \mathbf{U}_{0}(z, t) \xi \rightarrow 0 \quad|\xi| \rightarrow+\infty \\
\hat{p}(\xi) \rightarrow 0 \quad|\xi| \rightarrow+\infty .
\end{array}\right.
$$

Here $\mathbf{U}_{0}(x, t)$ is the background solution, that is, the solution in absence of any anomalies.

The minimization problem for reconstructing the shape and the shear modulus of the anomaly reads

$$
\min _{\tilde{\mu}, f} \frac{1}{2}\left\|\delta \mathbf{u}(x, t)-\epsilon \hat{\mathbf{v}}\left(\frac{x-z}{\epsilon}, t\right)+\nabla \mathbf{U}_{0}(z, t)(x-z)\right\|_{L^{2}(W \times[0, T])}^{2}+\beta \int_{W}|\nabla h(x)| d x,
$$

subject to $(7.2)$.

8. Conclusion. In this paper we have designed a new and promising way for reconstructing the shear modulus and the shape of a small elastic anomaly from internal measurements of displacement fields. Our approach combines the inverse problem techniques for small viscous anomalies and a binary level set algorithm. Its extension to detect anisotropic anomalies follows immediately. It is also expected that our method will allow an accurate reconstruction of the map of the mechanical coefficients, in particular in the presence of anomalies. Concerns about the robustness of our method, with respect to incomplete and noisy data, will be discussed in a forthcoming work. 


\section{REFERENCES}

[1] C. Alves and H. Ammari, Boundary integral formulae for the reconstruction of imperfections of small diameter in an elastic medium, SIAM J. Appl. Math. (2001), 62, 94-106. MR1857537 (2003a:35195)

[2] H. Ammari and H. Kang, High-order terms in the asymptotic expansions of the steady-state voltage potentials in the presence of inhomogeneities of small diameter, SIAM J. Math. Anal., 34 (2003), 1152-1166. MR2001663 (2004e:35027)

[3] H. Ammari and H. Kang, Reconstruction of Small Inhomogeneities from Boundary Measurements, Lecture Notes in Mathematics, Volume 1846, Springer-Verlag, Berlin, 2004. MR2168949 (2006k:35295)

[4] H. Ammari and H. Kang, Boundary layer techniques for solving the Helmholtz equation in the presence of small inhomogeneities, J. Math. Anal. Appl., 296 (2004), 190-208. MR2070502 (2005c:35054)

[5] H. Ammari and H. Kang, Reconstruction of elastic inclusions of small volume via dynamic measurements, Appl. Math. Opt., 54 (2006), 223-235. MR2239535 (2007f:74059)

[6] H. Ammari and H. Kang, Polarization and Moment Tensors with Applications to Inverse Problems and Effective Medium Theory, Applied Mathematical Sciences, Volume 162, Springer-Verlag, New York, 2007.

[7] H. Ammari, H. Kang, G. Nakamura, and K. Tanuma, Complete asymptotic expansions of solutions of the system of elastostatics in the presence of an inclusion of small diameter and detection of an inclusion, J. Elasticity, 67 (2002), 97-129. MR1985444 (2004c:74005)

[8] H. Ammari and A. Khelifi, Electromagnetic scattering by small dielectric inhomogeneities, J. Math. Pures Appl., 82 (2003), 749-842. MR2005296 (2005a:35262)

[9] H. Ammari, M. Vogelius and D. Volkov, Asymptotic formulas for perturbations in the electromagnetic fields due to the presence of inhomogeneities of small diameter II. The full Maxwell equations, J. Math. Pures Appl., 80 (2001), 769-814. MR1860816 (2003b:78002)

[10] P.M. Anselone, Collectively Compact Operator Approximation Theory and Applications to Integral Equations, Prentice-Hall, Englewood Cliffs, NJ, 1971. MR0443383 (56:1753)

[11] P.E. Barbone and J.C. Bamber, Quantitative elasticity imaging: what can and cannot be inferred from strain images, Phys. Med. Biol., 47 (2002), 2147-2164.

[12] E. Beretta, E. Francini and M. Vogelius, Asymptotic formulas for steady state voltage potentials in the presence of thin inhomogeneities. A rigorous error analysis, J. Math. Pures Appl., 82 (2003), 1277-1301. MR2020923 (2004i:35021)

[13] Y. Capdeboscq and H. Kang, Improved Hashin-Shtrikman bounds for elastic moment tensors and an application, preprint 2007.

[14] Y. Capdeboscq and M.S. Vogelius, Optimal asymptotic estimates for the volume of internal inhomogeneities in terms of multiple boundary measurements, Math. Modelling Num. Anal., 37 (2003), 227-240. MR1991198 (2005c:35067)

[15] D.J. Cedio-Fengya, S. Moskow and M. Vogelius, Identification of conductivity imperfections of small diameter by boundary measurements. Continuous dependence and computational reconstruction, Inverse Problems, 14 (1998), 553-595. MR1629995 (99d:78011)

[16] T.F. Chan and X.-C. Tai, Level set and total variation regularization for elliptic inverse problems with discontinuous coefficients, J. Comput. Phys., 193 (2003), 40-66. MR2022688 (2004j:65170)

[17] Z. Chen and J. Zou, An augmented Lagrangian method for identifying discontinuous parameters in elliptic systems, SIAM J. Cont. Opt., 37 (1999), 892-910. MR1680814 (2000d:65203)

[18] J.F. Douglas and A. Friedman, Coping with complex boundaries, IMA Series on Mathematics and its Applications, Vol. 67, 166-185, Springer, New York, 1995. MR1340194 (96f:00012)

[19] A. Friedman and M.S. Vogelius, Identification of small inhomogeneities of extreme conductivity by boundary measurements: a theorem on continuous dependence, Arch. Rat. Mech. Anal., 105 (1989), 299-326. MR973245 (90c:35198)

[20] J.F. Greenleaf, M. Fatemi, and M. Insana, Selected methods for imaging elastic properties of biological tissues, Annu. Rev. Biomed. Eng., 5 (2003), 57-78.

[21] L. Halpern, Spectral methods in polar coordinates for the Stokes problem. Application to computation in unbounded domains, Math. Comput., 65 (1996), 507-531. MR1333315 (97e:65100)

[22] L. Halpern, A spectral method for the Stokes problem in three-dimensional unbounded domains, Math. Comput., 70 (2001), 1417-1436. MR1836911 (2002d:76036)

[23] K. Ito, K. Kunisch, and Z. Li, Level-set function approach to an inverse interface problem, Inverse Problems, 17 (2001), 1225-1242. MR1862188 (2002h:35335) 
[24] L. Ji and J.R. McLaughlin, Recovery of the Lamé parameter $\mu$ in biological tissues, Inverse Problems, 20 (2004), 1-24. MR2044603 (2005b:74069)

[25] H. Kang, E. Kim, J.-Y. Lee, Identification of elastic inclusions and elastic moment tensors by boundary measurements, Inverse Problems, 19 (2003), 703-724. MR1984885 (2004c:74025)

[26] A. Kozhevnikov, On the first stationary boundary-value problem of elasticity in weighted Sobolev spaces in exterior domains of $\mathbb{R}^{3}$, Appl. Math. Opt., 34 (1996), 183-190. MR1397779 (97d:35217)

[27] O.A. Ladyzhenskaya, The Mathematical Theory of Viscous Incompressible Flow, Second English Edition, Gordon and Breach, New York, 1969. MR0254401 (40:7610)

[28] R. Lipton, Inequalities for electric and elastic polarization tensors with applications to random composites, J. Mech. Phys. Solids, 41 (1993), 809-833. MR1214019 (94d:73005)

[29] A. Manduca, T.E. Oliphant, M.A. Dresner, J.L. Mahowald, S.A. Kruse, E. Amromin, J.P. Felmlee, J.F. Greenleaf, and R.L. Ehman, Magnetic resonance elastography: Non-invasive mapping of tissue elasticity, Medical Image Analysis, 5 (2001), 237-254.

[30] J.R. McLaughlin and J.R. Yoon, Unique identifiability of elastic parameters from time-dependent interior displacement measurement, Inverse Problems, 20 (2004), 25-45. MR2044604 (2005b:74070)

[31] R. Muthupillai, D.J. Lomas, P.J. Rossman, J.F. Greenleaf, A. Manduca, and R.L. Ehman, Magnetic resonance elastography by direct visualization of propagating acoustic strain waves, Science, 269 (1995), 1854-1857.

[32] L.K. Nielsen, X.-C. Tai, S.I. Aanonsenzand, and M. Espedal, A binary level set model for elliptic inverse problems with discontinuous coefficients, Int. J. Numer. Anal. Model., 4 (2007), 74-99. MR2289734

[33] S. Osher and J.A. Sethian, Fronts propagating with curvature-dependent speed: algorithms based on Hamilton-Jacobi formulations, J. Comput. Phys., 79 (1988), 12-49. MR965860 (89h:80012)

[34] S. Ozawa, Singular variation of domains and eigenvalues of the Laplacian, Duke Math. J., 48 (1981), 767-778. MR782576 (86k:35117)

[35] R. Sinkus, J. Lorenzen, D. Schrader, M. Lorenzen, M. Dargatz, and D. Holz, High-resolution tensor MR elastography for breast tumour detection, Phys. Med. Biol., 45 (2000), 1649-1664.

[36] R. Sinkus, M. Tanter, S. Catheline, J. Lorenzen, C. Kuhl, E. Sondermann, and M. Fink, Imaging anisotropic and viscous properties of breast tissue by magnetic resonance-elastography, Mag. Res. Med., 53 (2005), 372-387.

[37] R. Sinkus, M. Tanter, T. Xydeas, S. Catheline, J. Bercoff, and M. Fink, Viscoelastic shear properties of in vivo breast lesions measured by MR elastography, Mag. Res. Imag., 23 (2005), 159-165.

[38] R. Temam, Navier-Stokes Equations, Theory and Numerical Analysis, North-Holland, Amsterdam, 1979. MR603444 (82b:35133)

[39] M. Vogelius and D. Volkov, Asymptotic formulas for perturbations in the electromagnetic fields due to the presence of inhomogeneities, Math. Model. Numer. Anal., 34 (2000), 723-748. MR1784483 (2001f:78024) 